Miguel Angel Orrillo Cumpa

\title{
Análise em Grassmannianas e o Teorema de Johnson-Lindenstrauss
}

Dissertação de Mestrado

Dissertação apresentada ao Programa de Pós-graduação em Matemática da PUC-Rio como requisito parcial para obtenção do grau de Mestre em Matemática.

Orientador: Prof. Carlos Tomei 


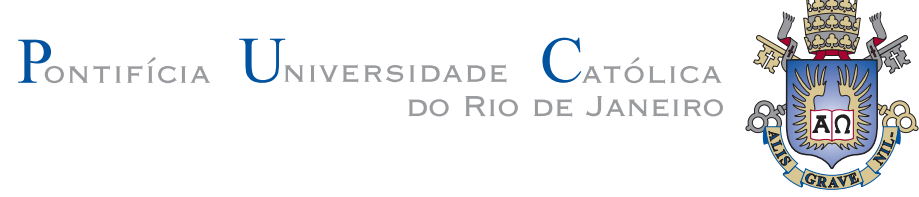

Miguel Angel Orrillo Cumpa

\title{
Análise em Grassmannianas e o Teorema de Johnson-Lindenstrauss
}

Dissertação apresentada ao Programa de Pós-graduação em Matemática da PUC-Rio como requisito parcial para obtenção do grau de Mestre em Matemática. Aprovada pela Comissão Examinadora abaixo assinada.

\author{
Prof. Carlos Tomei \\ Orientador \\ Departamento de Matemática — PUC-Rio \\ Prof. Nicolau Corção Saldanha \\ Departamento de Matemática — PUC-Rio
}

Prof. Juliana Freire

Departamento de Matemática — PUC-Rio

Prof. Roberto Imbuzeiro M.F. de Oliveira Instituto Nacional de Matemática Pura e Aplicada — IMPA

Prof. José Eugênio Leal Coordenador Setorial do Centro Técnico Científico - PUC-Rio 
Todos os direitos reservados. É proibida a reprodução total ou parcial do trabalho sem autorização da universidade, do autor e do orientador.

\section{Miguel Angel Orrillo Cumpa}

Bacharel em Matemática Pura - Universidade Federal Fluminense.

Ficha Catalográfica

Orrillo, Miguel

Análise em Grassmannianas e o Teorema de JohnsonLindenstrauss / Miguel Angel Orrillo Cumpa; orientador: Carlos Tomei. - Rio de Janeiro: PUC-Rio, Departamento de Matemática, 2013.

v., 63 f: il. ; $29,7 \mathrm{~cm}$

1. Dissertação (mestrado) - Pontifícia Universidade Católica do Rio de Janeiro, Departamento de Matemática.

Inclui referências bibliográficas.

1. Matemática - Dissertação. 2. Concentração de Medida. 3. Variedades de Grassmann. 4. Teorema de Johnson-Lindenstrauss. I. Tomei, Carlos. II. Pontifícia Universidade Católica do Rio de Janeiro. Departamento de Matemática. III. Título. 
A la memoria de Roxy Orrillo. 


\section{Agradecimentos}

Benedictus Dominus Deus Israel. A Deus pela graça concedida desta dissertação. A Ele e todos os seus santos.

A Jaime Orrillo, meu professor da vida toda, por ter-me mostrado sempre a matemática de uma forma mais aprazível. A ele por sua infinita persistência. A mi madre Yolanda por su amor, dedicación, educación y por la tenacidad en darme siempre todo su apoyo. A mis abuelos José, Rosa y Laura, por sus oraciones y pedidos constantes de protección. A mis hermanas Leslie y Roxana ( in memorian ) y a mi tía Juana por confiar en mi capacidad deseándome siempre lo mejor. A todos mis familiares en general, primos, primas, tíos y tías. A todos ellos por el cariño que siempre me dieron y que hicieron, aún estando lejos, sentir la confianza de pertenecer a una familia grande, unida y querida. Um agradecimento mais do que especial à minha companheira e esposa Amanda Orrillo pelo amor, pela compreensão e apoio que foram essências nesses momentos de dissertação. À minha querida sogra Serly Soares pelo papel de segunda mãe que ela desempenhou durante nossa convivência.

Ao meu orientador Carlos Tomei pela disponibilidade, entusiasmo e principalmente por me outorgar um pouco da sua erudição. Aos membros da banca: Nicolau Corção Saldanha, Roberto Imbuzeiro e Juliana Freire por aceitar e revisar a minha dissertação. Aos professores Jairo Bochi, Thomas Lewiner, Ricardo Sá Earp, Alex Castro, meus professores durante o mestrado na Puc-Rio. A eles muito obrigado pela formação.

À Pontifícia Universidade Católica do Rio de Janeiro pela bolsa de isensão e sua magnífica estrutura. Ao departamento de matemática da PUC-Rio e sua funcionária Creuza Nascimento pelo zelo e pelas constantes exortações.

Aos meus queridos amigos do peito, irmãos, Acir Carlos da Silva Junior, Johel Beltrán, João Marcos Breia Jucá, Juan Pablo Luna y Victor Goulart Nascimento pela leitura, correção ou sugestões outorgadas durante toda a realização desta dissertação. Aos meus queridos amigos Felipe Melo, Rodrigo Pacheco, Cong Zhou, Américo Cunha e André Zaccur pelas dicas de latex e gráficas. Um agradecimento especial ao meu querido amigo do peito Eric Biagioli pela organização "latexiana" da minha dissertação. Muito obrigado a todos os outros meus amigos que, felizmente sendo muitos, não acabaria de mencioná-los aqui. De uma maneira especial, ao meu querido padrinho espiritual Carlos Eduardo Guedes Belchior (Cadú). Um obrigado especialíssimo à minha querida Hazel Crato, ao meu amigo irmão de fé Juan Eduardo Casavilca, meu amigo Guillermo Gomez e à família Jucá (Débora, Celso, Pedro e Tche). A todos eles pela sincera torcida. Ao meu amigo Maycol junto com Carolina Parra (pelos '5' inegáveis) e seus familiares. Ao meu querido 
"compadre" Daniel por sua amizade sincera e suas irreverências. A todos eles, um muito obrigado por fazer parte desta minha segunda família, meus amigos.

Finalmente, à Coordenação de Aperfeiçoamento de Pessoal de Nível Superior (CAPES) pela bolsa concedida. 


\section{Resumo}

Orrillo, Miguel; Tomei, Carlos. Análise em Grassmannianas e o Teorema de Johnson-Lindenstrauss. Rio de Janeiro, 2013. 63p. Dissertação de Mestrado - Departamento de Matemática, Pontifícia Universidade Católica do Rio de Janeiro.

Seja V um conjunto de $\mathrm{n}$ pontos no espaço euclidiano $\mathrm{X}$ de dimensão d. Pelo teorema de Johnson-Lindenstrauss, existe uma projeção entre X e $\mathrm{Y}$, outro espaço de dimensão $\mathrm{k}$ bastante menor, com a propriedade que as distâncias entre imagens de pontos de $\mathrm{V}$ sejam mantidas dentro de um fator c arbitrariamente próximo de 1. O teorema apresenta uma relação entre d, k e c, indicando a possibilidade de dramáticas reduções de dimensão para representações fidedignas de V. A demonstração emprega as Grassmannianas, as variedades de subespaços de dimensão k em X. São construídas cartas e uma medida homogênea em relação à ação natural do grupo ortogonal na Grassmanniana. O resultado segue estimando através de gaussianas certas integrais de caráter fortemente geométrico.

\section{Palavras-chave}

Concentração de Medida; Variedades de Grassmann; Teorema de Johnson-Lindenstrauss; 


\section{Abstract}

Orrillo, Miguel; Tomei, Carlos (advisor) . Grassmanian Analysis and the Johnson-Lindenstrauss Theorem.. Rio de Janeiro, 2013. 63p. M.Sc. Dissertation — Departamento de Matemática, Pontifícia Universidade Católica do Rio de Janeiro.

Let $\mathrm{V}$ be a set of $\mathrm{n}$ points in the Euclidean space $\mathrm{X}$ of dimension d. The Johnson-Lindenstrauss theorem states that there is a projection between $\mathrm{X}$ a and $\mathrm{Y}$, another Euclidean space of a smaller dimension $\mathrm{k}$, with the property that images of points of $\mathrm{X}$ under projection do not differ by more that a multiplicative factor c arbitrarily close to 1 . The theorem presents a relation among $\mathrm{d}, \mathrm{k}$ and $\mathrm{c}$, indicating the possibility of dramatic dimensional reduction of very faithful representations of $\mathrm{V}$. The proof makes use of Grassmanians, the manifolds consisting of subspaces of dimension $\mathrm{k}$ in X. In the text, charts are presented, together with a measure which is homogeneous with respect to the natural action of the orthogonal group on the Grassmanian. The result follows by taking estimates using gaussians of certain integrals with a strong geometric flavor.

\section{Keywords}

Measure Concentration; Grassmann Manifolds; Johnson-Lindenstrauss Theorem; 


\section{Sumário}

1 Introdução 12

2 Preliminares $\quad 15$

3 Concentração de Medida na Esfera $\quad 18$

$\begin{array}{ll}3.1 \text { A função gama } & 18\end{array}$

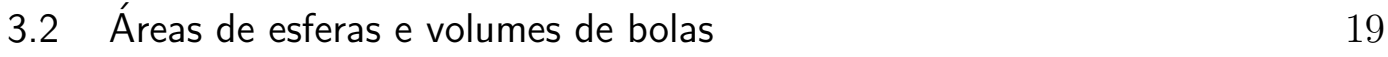

3.3 Coordenadas esféricas e o elemento de área de $S^{d-1} \quad 21$

3.4 Concentração em faixas equatoriais 24

4 Análise em Grassmannianas $\quad 28$

4.1 $G_{k}\left(\mathbb{R}^{d}\right)$ como subvariedade de $\mathcal{S}\left(\mathbb{R}^{d}\right) \quad 29$

$\begin{array}{lll}4.2 & \text { A função posto } & 30\end{array}$

$\begin{array}{lll}4.3 & G_{k}\left(\mathbb{R}^{d}\right) \text { como variedade diferenciável } & 37\end{array}$

$5 \quad$ O Teorema de Johnson-Lindenstrauss $\quad 43$

5.1 Medida de Haar em $\mathcal{O}(d) \quad 43$

5.2 Medidas invariantes em $S^{d-1}$ e em $G_{k}\left(\mathbb{R}^{d}\right) \quad 46$

5.3 Gaussianas e o lema geométrico 47

5.4 Demonstração do teorema de Johnson-Lindenstrauss 55

$\begin{array}{ll}\text { A Noções de Variedades } & 58\end{array}$

$\begin{array}{ll}\text { B Noções de Probabilidade } & 60\end{array}$

Referências Bibliográficas $\quad 62$ 


\section{Lista de Figuras}

1.1 Faixa equatorial de espessura $h$ subtendendo um ângulo $\alpha$. $\quad 12$

1.2 Área coberta por uma faixa de espessura $h$. 12

$1.3 C_{1}, C_{2} \subset S^{2}$ representam os pontos da esfera com $\left|x_{1}\right| \geq t$. $\quad 13$

3.1 Coordenadas esféricas em $d=3$. 22

3.2 O gráfico representa a desigualdade $\cos (x) \leq \exp \left(-x^{2} / 2\right)$. 26 
Mesmo que eu tivesse o dom da profecia, e conhecesse todos os mistérios e toda a ciência; mesmo que tivesse toda a fé, a ponto de transportar montanhas, se não tiver caridade, não sou nada.

1 Coríntios, 13:2. 


\section{Introdução}

Suponha que você queira pintar a superfície da Terra de raio $\rho$. Para isso você conta com a ajuda de um rolo capaz de pintar faixas gigantes ao redor da Terra. Que fração $x$ da área da esfera você terá coberto depois de passar o rolo ao redor do equador pintando assim uma faixa de espessura $h$ subtendendo um ângulo $\alpha$ ?

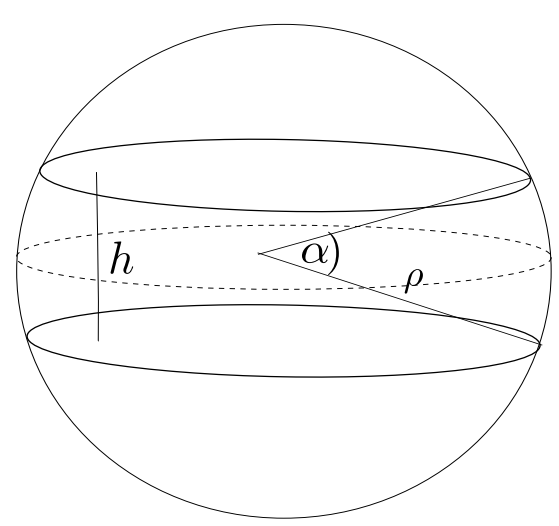

Figura 1.1: Faixa equatorial de espessura $h$ subtendendo um ângulo $\alpha$.

O problema é resolvido usando o seguinte fato geral:

$N a$ esfera de raio $\rho$, a área de uma faixa qualquer de espessura $h$ é $2 \pi \rho h$.

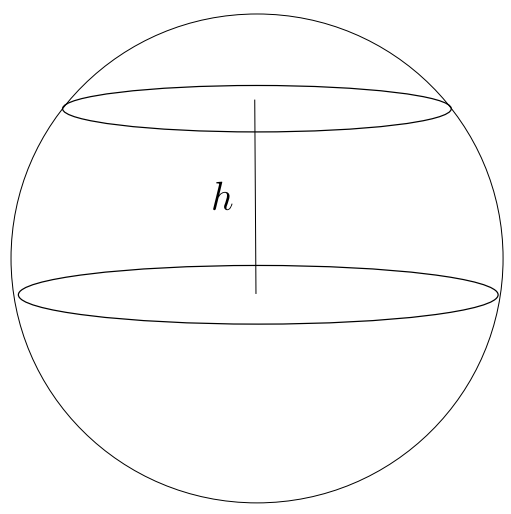

Figura 1.2: Área coberta por uma faixa de espessura $h$. 
Dado que faixa tem espessura $h$ e subtende um ângulo $\alpha$, então $\operatorname{sen}(\alpha / 2)=h / 2 \rho$. Logo, uma simples regra de três permite calcular a porcentagem

$$
x=\frac{2 \pi \rho h}{4 \pi \rho^{2}} 100=\frac{h}{2 \rho} 100=\operatorname{sen}\left(\frac{\alpha}{2}\right) 100 .
$$

Por exemplo, se $\alpha=10^{\circ}$ (cuja faixa tem mais de $1000 \mathrm{Km}$ de largura), então a fração pintada seria aproximadamente $8,7 \%$.

O fenômeno de concentração de medida garante que sua tarefa seria muito mais simples se a Terra fosse de dimensão alta. Mais especificamente, uma só volta à Terra com o rolo ao redor do equador seria suficiente para cobrir praticamente toda sua superfície. Isto significa que, para $d$ muito grande, a área da superfície da esfera $S^{d-1}=\left\{x \in \mathbb{R}^{d}:\|x\|=1\right\}$ é concentrada em torno do equador. Ou seja, a área do complemento da faixa equatorial (duas calotas esféricas) tende a 0 quando $d$ tende ao infinito.

O teorema de Johnson-Lindenstrauss é o resultado principal desta dissertação. Ele mostra que a geometria de um conjunto $V$ com $n$ pontos não é muito perturbada por certas projeções ortogonais sobre subespaços de dimensão logarítmica de $n$. Em outras palavras, é possível projetar $V$ em subespaços de dimensão baixa preservando bem a distância entre eles.

A prova usa um lema geométrico relacionado com a concentração da área em faixas equatoriais. Tome $s=\left(x_{1}, \cdots, x_{d}\right)$ um ponto de $S^{d-1}$ escolhido uniformemente e seja $\pi_{\mathbb{R}^{k}}(s)=\left(x_{1}, \cdots, x_{k}\right)$ a projeção de $s$ em suas $k$ primeiras coordenadas - projeção ortogonal em $\mathbb{R}^{k}$. Considere $\beta$ um número próximo de 1 de modo que $t=\sqrt{\beta \frac{k}{d}}<1$. Qual seria a probabilidade de $\left\|\pi_{\mathbb{R}^{k}}(s)\right\|$ ser maior ou igual que $t$ ? Quando $k=1$ e $d=3$, a geometria fica clara na seguinte figura.

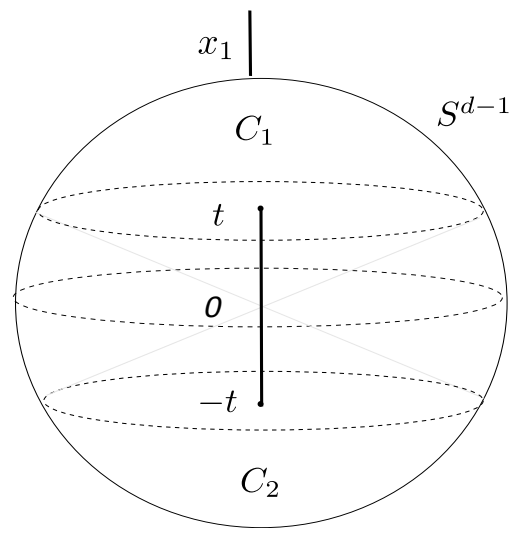

Figura 1.3: $C_{1}, C_{2} \subset S^{2}$ representam os pontos da esfera com $\left|x_{1}\right| \geq t$.

Observamos que $\operatorname{Pr}\left[\left|x_{1}\right|>\sqrt{\beta \frac{k}{d}}\right]=1-x$, onde $x$ é a fração de área da 
esfera na faixa de espessura $t$. Quando $d$ tende ao infinito, pela concentração de medida em faixas equatoriais, concluiríamos que $\operatorname{Pr}\left[\left|x_{1}\right|>t\right]$ tende a 0 .

Um resultado mais geral vale para $k<d$ qualquer - para uma melhor descrição, o leitor pode consultar o Lema 5.6 do Capítulo 5.- Essencialmente, o lema afirma que o quadrado da norma da projeção em $\mathbb{R}^{k}$ de pontos da esfera $S^{d-1}$, escolhidos uniformemente, se encontra fortemente concentrado em torno de sua média $k / d$.

A afirmação acima tem outra interpretação: em vez de variar o ponto e fixar uma projeção ortogonal de imagem com dimensão $k$, podemos fixar o ponto e fazer a média sobre todas as projeções ortogonais possíveis com imagem de dimensão $k$. A prova do teorema emprega o fato de que a norma de ambas as projeções possuem a mesma distribuição. 


\section{2 \\ Preliminares}

Neste capítulo coletaremos alguns fatos básicos. Todas as definições e resultados aqui apresentados são bem conhecidos e podem ser encontrados na literatura.

Supomos que o leitor tenha familiaridade com os conceitos de espaços topológicos, métricos e de medida, grupos e subgrupos e alguns conceitos e resultados básicos de álgebra linear, tais como projeções ortogonais, o teorema espectral, processo de ortogonalização de Gram-Schmidt, etc. Para o vocabulário básico sobre variedades diferenciáveis e de probabilidade, o leitor pode considerar os apêndices.

Alguns conjuntos de matrizes serão usados extensivamente. Seja $\mathcal{M}(d, \mathbb{R})$ o conjunto de todas as matrizes reais $d \times d$ com a norma de Frobenius

$$
\|A\|_{2}=\left(\sum_{i=1}^{d} \sum_{j=1}^{d} a_{i j}^{2}\right)^{1 / 2}, \quad A=\left(a_{i j}\right) .
$$

O grupo linear formado por todas as matrizes inversíveis $d \times d$

$$
G L\left(\mathbb{R}^{d}\right)=\{A \in \mathcal{M}(d, \mathbb{R}): \operatorname{det}(A) \neq 0\}
$$

é um subconjunto aberto de $\mathcal{M}(d, \mathbb{R})$. O grupo ortogonal $\mathcal{O}(d)$ é um subgrupo de $G L\left(\mathbb{R}^{d}\right)$, definido como

$$
\mathcal{O}(d)=\left\{Q \in G L\left(\mathbb{R}^{d}\right): Q^{*} Q=Q Q^{*}=I\right\}
$$

Além disso, $\mathcal{O}(d)$ é uma subvariedade compacta suave $\left(C^{\infty}\right)$ de dimensão $d(d-1) / 2$.

A esfera $S^{d-1}=\left\{x \in \mathbb{R}^{d}:\|x\|^{2}=\sum_{i=1}^{n} x_{i}^{2}=1\right\}$ é uma subvariedade compacta suave de codimensão 1 e classe $C^{\infty}$.

Um grupo $G$ é um grupo topológico quando $G$ é um espaço topológico e as operações de multiplicação e de tomada de inverso, $(a, b) \mapsto a b$ e $a \mapsto a^{-1}$, são contínuas.

Seja $M$ uma variedade diferenciável e $G$ um grupo topológico. Uma ação 
de $G$ sobre $M$ é uma aplicação contínua $\phi: G \times M \rightarrow M$ tal que

(i) Se $e$ é o elemento neutro de $G$ então $\phi(e, x)=x, \forall x \in M$

(ii) $\phi(g, \phi(h, x))=\phi(g h, x)$ para todo $g, h \in G$ e para todo $x \in M$.

A ação $\phi: G \times M \rightarrow M$ é transitiva, ou $\mathrm{G}$ age transitivamente em $M$ através de $\phi$, se para todo $x, y \in M$, existe $g \in G$ tal que $\phi(g, x)=y$. Nesse texto, vamos usar a ação do grupo ortogonal $G=\mathcal{O}(d)$ sobre a esfera $M=S^{d-1}$, dada por

$$
\begin{aligned}
\phi: \mathcal{O}(d) \times S^{d-1} & \rightarrow S^{d-1} \\
(g, x) & \mapsto g x .
\end{aligned}
$$

Seja $\phi$ uma ação de um grupo $G$ sobre uma variedade $M$ e $x \in M$. A $\phi$-órbita de $x$ é por definição o conjunto $G x=\{\phi(g, x): g \in G\}$.

A ação $\phi: G \times M \rightarrow M$ é própria se e somente se $\tilde{\phi}: G \times M \rightarrow M \times M$ definida por $\tilde{\phi}(g, x)=(x, \phi(g, x))$ é uma aplicação própria, isto é, inversa de compacto é compacto. A demonstração da seguinte proposição pode ser encontrada em (1, p. 265).

Proposição 2.0.1 Seja $\phi: G \times M \rightarrow M$ uma ação própria. Então toda órbita Gx é uma subvariedade fechada de $M$.

Lembramos ao leitor que em um espaço topológico $X$ a $\sigma$-álgebra de Borel é a menor $\sigma$-álgebra que contém todos os abertos de $X$. Seus elementos são os borelianos de $X$.

Funções transferem medidas de um espaço para outro através do pushforward. Mais especificamente, sejam $(X, \mathfrak{X}, \mu)$ um espaço de medida, $Y$ um espaço topológico qualquer e $\phi: X \rightarrow Y$ uma aplicação. A medida induzida $\phi_{*} \mu$ é definida para todo boreliano $B$ de $Y$ como

$$
\phi_{*} \mu(B):=\mu\left(\phi^{-1}(B)\right)=\mu(\{g \in G: \phi(g) \in B\}) .
$$

Uma medida $\nu$ de $M$ é invariante pela ação $\phi: G \times M \rightarrow M$ se para todo $g \in G$ e para todo conjunto boreliano $A \subset M$, vale

$$
\nu(A)=\nu(g A)=\nu(\{\phi(g, a): a \in A\}) .
$$

$\operatorname{Sejam~} \mathcal{B}\left(\mathbb{R}^{d}\right)$ a $\sigma$-álgebra de Borel em $\mathbb{R}^{d}$ e $\lambda$ a medida de Lebesgue. Uma ação do grupo ortogonal $\mathcal{O}(d)$ sobre $\mathbb{R}^{d}$ é naturalmente dada por

$$
\phi: \mathcal{O}(d) \times \mathbb{R}^{d} \rightarrow \mathbb{R}^{d}, \quad \phi(g, x)=g x
$$


Terminamos o capítulo mostrando que a medida de Lebesgue é invariante por essa ação.

Lembramos ao leitor que toda transformação linear $T: \mathbb{R}^{d} \rightarrow \mathbb{R}^{d}$ é o produto de transformações elementares, dadas por

$$
\begin{aligned}
T_{1}\left(x_{1}, \cdots, x_{i}, \cdots, x_{j}, \cdots, x_{d}\right) & =\left(x_{1}, \cdots, x_{j}, \cdots, x_{i}, \cdots, x_{d}\right) \\
T_{2}\left(x_{1}, \cdots, x_{i}, \cdots, x_{d}\right) & =\left(x_{1}, \cdots, c x_{i}, \cdots, x_{d}\right) \quad(c \neq 0) \\
T_{3}\left(x_{1}, \cdots, x_{i}, \cdots, x_{k}, \cdots, x_{d}\right) & =\left(x_{1}, \cdots, x_{i}+c x_{k}, \cdots, x_{k}, \cdots, x_{d}\right)(k \neq i) .
\end{aligned}
$$

Proposição 2.0.2 Seja $T: \mathbb{R}^{d} \rightarrow \mathbb{R}^{d}$ uma transformação linear inversível $e$ $A \subset \mathbb{R}^{d}$ um boreliano. Então

$$
\lambda(T(A))=|\operatorname{det}(T)| \lambda(A)
$$

Demostração: Suponha que a proposição seja verdadeira para transformações $T$ e $S$. Então ela vale para $T \circ S$

$\lambda((T \circ S)(A))=|\operatorname{det}(T)| \lambda(S(A))=|\operatorname{det}(T)||\operatorname{det}(S)| \lambda(A)=|\operatorname{det}(T \circ S)| \lambda(A)$.

Logo, é suficiente mostrar que a proposição é valida para as transformações elementares $T_{1}, T_{2}$ e $T_{3}$. Explicitamos o argumento para $T_{3}$ onde $\operatorname{det}\left(T_{3}\right)=1$. Usamos Fubini afim de trocar a ordem de integração entre $x_{i}$ e $x_{j}$.

$$
\begin{aligned}
\left|\operatorname{det}\left(T_{3}\right)\right| \lambda(A) & =\lambda(A)=\int_{A} 1 d x_{1} \cdots d x_{i} \cdots d x_{j} \cdots d x_{d} \\
& =\int_{A} 1 d x_{1} \cdots d x_{j} \cdots d x_{i} \cdots d x_{d} \\
& =\int_{T_{3}(A)} 1 d x_{1} \cdots d x_{i} \cdots d x_{j} \cdots d x_{d}=\lambda\left(T_{3}(A)\right) .
\end{aligned}
$$

Corolário 2.1 A medida de Lebesgue é invariante pela ação do grupo ortogonal.

Demostração: Transformações lineares $g \in \mathcal{O}(d)$ satisfazem $g g^{*}=I$, o que implica que $|\operatorname{det}(g)|=1$. Logo, $\lambda(g(A))=\lambda(A)$. 


\section{Concentração de Medida na Esfera}

Um primeiro exemplo do fenômeno de concentração de medida ocorre na esfera $S^{d-1} \subset \mathbb{R}^{d}$. Este capítulo tem o propósito de apresentá-lo detalhadamente: a medida uniforme da esfera se concentra em faixas equatoriais quando a dimensão tende ao infinito - para uma descrição precisa, o leitor pode consultar a equação (3-5).

Começamos introduzindo a função gama e algumas contas básicas: o cálculo das áreas das esferas e do volume das bolas na métrica euclidiana em $\mathbb{R}^{d}$, cujas fórmulas envolvem a função gama. Finalmente, mostraremos a concentração da esfera em faixas equatoriais. Aqui, usaremos coordenadas esféricas para calcular a área de calotas esféricas.

\section{1}

\section{A função gama}

A função gama é

$$
\Gamma(z)=\int_{0}^{\infty} t^{z-1} \exp (-t) d t, \quad \text { para } z \in \mathbb{C}, \operatorname{Re}(z)>0
$$

Para conveniência do leitor, mostramos sua integrabilidade.

Proposição 3.1.1 A função gama é bem definida.

Demonstração: $\mathrm{O}$ argumento é diferente para $t$ perto e longe de 0

$$
\int_{0}^{\infty}\left|t^{z-1} \exp (-t)\right| d t=\int_{0}^{1}\left|t^{z-1} \exp (-t)\right| d t+\int_{1}^{\infty}\left|t^{z-1} \exp (-t)\right| d t
$$

Para a primeira parcela, como $t \geq 0$, temos $\exp (-t) \leq 1$ e assim

$$
\begin{aligned}
\left|t^{z-1} \exp (-t)\right| & \leq\left|t^{z-1}\right|=|\exp ((z-1) \log t)| \\
& =\exp ((\operatorname{Re}(z)-1) \log t)=\exp \left(\log t^{\operatorname{Re}(z)-1}\right)=t^{(\operatorname{Re}(z)-1)} .
\end{aligned}
$$

Como $\int_{0}^{1} t^{a}<\infty$ para todo $a>-1$,

$$
\int_{0}^{1}\left|t^{z-1} \exp (-t)\right| d t \leq \int_{0}^{1} t^{\operatorname{Re}(z)-1}<\infty .
$$


Para a segunda parcela, $t \geq 1$ e temos

$$
\left|t^{z-1} \exp (-t)\right|=t^{\operatorname{Re}(z)-1} \exp (-t)
$$

A função $h(t)=t^{\operatorname{Re}(z)-1} \exp (-t / 2)$ é contínua em $[1, \infty)$ e $\lim _{t \rightarrow \infty} h(t)=0$, logo $h$ é limitada, $|h| \leq C_{z}$. Assim,

$$
\left|t^{z-1} \exp (-t)\right|=t^{\operatorname{Re}(z)-1} \exp (-t) \leq C_{z} \exp (-t / 2)
$$

Como $\int_{1}^{\infty} C_{z} \exp (-t / 2)<\infty$, a segunda parcela também é integrável.

Integrando por partes,

$\int_{0}^{\infty} t^{z} \exp (-t) d t=-\left.t^{z} \exp (-t)\right|_{0} ^{\infty}+z \int_{0}^{\infty} t^{z-1} \exp (-t) d t=z \int_{0}^{\infty} t^{z-1} \exp (-t) d t$

concluímos que para $\operatorname{Re}(z)>0$ a função gama satisfaz a conhecida equação funcional $\Gamma(z+1)=z \Gamma(z)$. Isto permite estendê-la para quase todos os números complexos. Além disso, por indução,

$$
\Gamma(n+1)=n ! \quad \text { e } \quad \Gamma\left(k+\frac{1}{2}\right)=\frac{\sqrt{\pi}(2 k-1) ! !}{2^{k}} .
$$

O fatorial duplo $(2 k-1)$ !! é dado pelo produto de todos os naturais ímpares até $(2 k-1)$.

\section{2}

\section{Áreas de esferas e volumes de bolas}

Indicamos por $S_{a}^{d-1}(R)$ e $B_{a}^{d}(R)$ respectivamente a esfera e a bola em $\mathbb{R}^{d}$ de raio $R$ centradas em $a \in \mathbb{R}^{d}$,

$$
S_{a}^{d-1}(R)=\left\{x \in \mathbb{R}^{d}:\|x-a\|=R\right\}, \quad B_{a}^{d}(R)=\left\{x \in \mathbb{R}^{d}:\|x-a\| \leq R\right\} .
$$

Quando $R=1$ e $a=0$, escreveremos simplesmente $S^{d-1}$ e $B^{d}$.

Agora definiremos a medida de Lebesgue na esfera $S^{d-1}$. Lembramos ao leitor que os borelianos da esfera $S_{0}^{d-1}(R)$ são todas as interseções da própria esfera com os borelianos de $\mathbb{R}^{d}$. Se $A \subset S_{0}^{d-1}(R)$ é um boreliano da esfera, denotemos por $C(A)$ o conjunto mensurável de $\mathbb{R}^{d}$ dado por $C(A)=\{r x: r \in$ $[0,1], x \in A\}$. Definimos, então, a medida de Lebesgue $\eta(A)$ de $A \in \mathcal{B}\left(S_{R}^{d-1}\right)$ como sendo

$$
\eta(A)=\frac{d}{R} \lambda(C(A))
$$


Fazendo $R=1,\left(S^{d-1}, \mathcal{B}\left(S^{d-1}\right), \eta\right)$ é um espaço de medida.

Proposição 3.2.1 A medida de Lebesgue $\eta$ em $S^{d-1}$ é invariante pela ação do grupo ortogonal $\mathcal{O}(d)$.

Demonstração: Dado que a medida de Lebesgue em $\mathbb{R}^{d}$ é invariante pela ação do grupo $\mathcal{O}(d) \cdot{ }^{1}$, a demonstração é direta. Observamos primeiro que se $g \in \mathcal{O}(d)$ e $A \in \mathcal{B}\left(S^{d-1}\right)$ então certamente $g C(A)=C(g A)$. Assim,

$$
\eta(A)=d \lambda(C(A))=d \lambda(g C(A))=d \lambda(C(g A))=\eta(g A) .
$$

A medida $\eta$ definida em $\mathcal{B}\left(S^{d-1}\right)$ é o análogo à medida que generaliza o comprimento em $S^{1}$ assim como $\lambda$ é o análogo a área em $\mathbb{R}^{2}$. Em $\mathbb{R}^{3}$, temos $S^{2}=\left\{x \in \mathbb{R}^{3}:\|x\|=1\right\}$ como fronteira de $B^{3}=\{x \in$ $\mathbb{R}^{3}$ : $\left.\|x\| \leq 1\right\}$. Neste caso, $\eta$ é o que conhecemos por área (na esfera) e $\lambda$ como volume.

\section{Proposição 3.2 .2}

$$
\lambda\left(B_{0}^{d}(R)\right)=\frac{\pi^{d / 2}}{\Gamma\left(\frac{d}{2}+1\right)} R^{d}, \quad \eta\left(S_{0}^{d-1}(R)\right)=\frac{2 \pi^{d / 2}}{\Gamma\left(\frac{d}{2}\right)} R^{d-1} .
$$

Demonstração: Primeiro mostraremos que

$$
\lambda\left(B_{0}^{d}(R)\right)= \begin{cases}\frac{\pi^{d / 2}}{\left(\frac{d}{2}\right) !} R^{d} & , \text { se } d \text { é par } ; \\ \frac{2^{(d+1) / 2} \pi^{(d-1) / 2}}{d ! !} R^{d} & , \text { se } d \text { é impar. }\end{cases}
$$

Denotemos como $V_{d}(R)=\lambda\left(B_{0}^{d}(R)\right)$. A geometria fica mais clara para $d=3$. Usando coordenadas polares, integramos no disco de raio $R$, onde para cada ponto $p$ temos uma bola de dimensão 1 (intervalo) de centro $p$. Assim,

$$
V_{3}(R)=\int_{0}^{R} \int_{0}^{2 \pi} V_{1}\left(\sqrt{R^{2}-r^{2}}\right) r d \theta d r=\int_{0}^{2 \pi} \int_{0}^{R}\left(2 \sqrt{R^{2}-r^{2}}\right) r d r d \theta
$$

e, fazendo a mudança de variável $u=R^{2}-r^{2}$,

$$
V_{3}(R)=\int_{0}^{2 \pi} 1 d \theta \int_{R^{2}}^{0}-u^{1 / 2} d u=2 \pi \int_{0}^{R^{2}} u^{1 / 2} d u=2 \pi\left(\left.\frac{2 u^{3 / 2}}{3}\right|_{0} ^{R^{2}}\right)=\frac{4 \pi}{3} R^{3} .
$$

Seja $T: \mathbb{R}^{d} \rightarrow \mathbb{R}^{d}, T(x)=R x$. Então aplicando a Proposição 2.0.2, é fácil calcular o volume de uma bola de raio $R$ a partir da bola unitária:

${ }^{1}$ Veja o Corolário 2.1 no capítulo 2. 
$V_{d}(R)=R^{d} V_{d}(1)$. Assim, obtemos a seguinte relação de recorrência

$$
\begin{aligned}
V_{d}(R) & =\int_{0}^{R} \int_{0}^{2 \pi} V_{d-2}\left(\sqrt{R^{2}-r^{2}}\right) r d \theta d r \\
& =\int_{0}^{2 \pi} \int_{0}^{R}\left(R^{2}-r^{2}\right)^{(d-2) / 2} V_{d-2}(1) r d r d \theta=\pi V_{d-2}(1) \int_{0}^{R^{2}} u^{(d-2) / 2} d u \\
& =\left.2 \pi V_{d-2}(1) \frac{u^{d / 2}}{d}\right|_{0} ^{R^{2}}=2 \pi V_{d-2}(1) \frac{R^{d}}{d}=\frac{2 \pi R^{2}}{d} V_{d-2}(R) .
\end{aligned}
$$

Agora usamos indução em $d$ para verificar o caso geral. Para $d=1,2$ temos $V_{1}(R)=2 R$ e $V_{2}(R)=\pi R^{2}$, de acordo com a fórmula (3-3).

Supondo a equação (3-3) válida para $d$ par, vamos verificar a sua validade para $d+2$ usando a fórmula $V_{d}(R)=\frac{2 \pi R^{2}}{d} V_{d-2}(R)$

$$
V_{d+2}(R)=\frac{2 \pi R^{2}}{d+2} V_{d}(R)=\frac{2 \pi R^{2}}{d+2} \frac{\pi^{d / 2}}{\left(\frac{d}{2}\right) !} R^{d}=\frac{2 \pi^{(d+2) / 2}}{2\left(\frac{d+2}{2}\right)\left(\frac{d}{2}\right) !}=\frac{\pi^{(d+2) / 2}}{\left(\frac{d+2}{2}\right) !} .
$$

Podemos fazer o mesmo para $d$ ímpar

$$
V_{d+2}(R)=\frac{2 \pi R^{2}}{d+2} V_{d}(R)=\frac{2 \pi R^{2}}{d+2} \frac{2^{(d+1) / 2} \pi^{(d-1) / 2}}{(d) ! !} R^{d}=\frac{2^{((d+2)+1) / 2} \pi^{((d+2)-1) / 2}}{(d+2) ! !} R^{d+2}
$$

Agora verificaremos a fórmula envolvendo a função gama. Com efeito, para $d=2 k$,

$$
\frac{\pi^{2 k / 2}}{\Gamma\left(\frac{2 k}{2}+1\right)} R^{2 k}=\frac{\pi^{k}}{\Gamma(k+1)} R^{2 k}=\frac{\pi^{k}}{k !} R^{2 k}=V_{2 k}(R)
$$

e para $d=2 k+1$

$$
\frac{\pi^{(2 k+1) / 2}}{\Gamma\left(\frac{2 k+1}{2}+1\right)} R^{2 k+1}=\frac{\pi^{(2 k+1) / 2}}{\frac{2 k+1}{2} \Gamma\left(k+\frac{1}{2}\right)} R^{2 k+1}=\frac{\pi^{k} 2^{k+1}}{(2 k+1) ! !} R^{2 k+1}=V_{2 k+1}(R) .
$$

Assim, concluímos que $\lambda\left(B_{0}^{d}(R)\right)=\frac{\pi^{d / 2}}{\Gamma\left(\frac{d}{2}+1\right)} R^{d}$, para todo $d$. Da definição, $\eta(A)=\frac{d}{R} \lambda(C(A))$, concluímos também

$$
\eta\left(S_{0}^{d-1}(R)\right)=\frac{d}{R} V_{d}(R)=\frac{d}{R} \frac{\pi^{d / 2}}{\Gamma\left(\frac{d}{2}+1\right)} R^{d}=d \frac{\pi^{d / 2}}{\frac{d}{2} \Gamma\left(\frac{d}{2}\right)} R^{d-1}=\frac{2 \pi^{d / 2}}{\Gamma\left(\frac{d}{2}\right)} R^{d-1} .
$$

\section{3}

Coordenadas esféricas e o elemento de área de $S^{d-1}$

Sejam $U, V \subset \mathbb{R}^{d}$ abertos, $\psi: U \rightarrow V$ um difeomorfismo $C^{1}$ e $X \subset U$ mensurável com $\psi(X) \subset V$ também mensurável. Considere $f: \psi(X) \rightarrow \mathbb{R}$ 
uma função integrável. Pelo teorema da mudança de variáveis,

$$
\int_{\psi(X)} f(y) d y=\int_{X} f(\psi(x)) \cdot|\operatorname{det} D \psi(x)| d x .
$$

A função $\psi$ é o que chamamos de mudança de variável. Veja (10). Entre os exemplos estão as mudanças de coordenadas tradicionais. As coordenadas esféricas consistem de uma coordenada radial $\rho \geq 0$ e ângulos $\phi_{1}, \phi_{2}, \ldots, \phi_{d-2} \in[0, \pi]$ e $\phi_{d-1} \in[0,2 \pi)$, definidos pelas fórmulas

$$
\begin{aligned}
& x_{1}=\rho \cos \left(\phi_{1}\right) \\
& x_{2}=\rho \operatorname{sen}\left(\phi_{1}\right) \cos \left(\phi_{2}\right) \\
& x_{3}=\rho \operatorname{sen}\left(\phi_{1}\right) \operatorname{sen}\left(\phi_{2}\right) \cos \left(\phi_{3}\right) \\
& \vdots \\
& x_{d-1}=\rho \operatorname{sen}\left(\phi_{1}\right) \cdots \operatorname{sen}\left(\phi_{d-2}\right) \cos \left(\phi_{d-1}\right) \\
& x_{d}=\rho \operatorname{sen}\left(\phi_{1}\right) \cdots \operatorname{sen}\left(\phi_{d-2}\right) \operatorname{sen}\left(\phi_{d-1}\right) .
\end{aligned}
$$

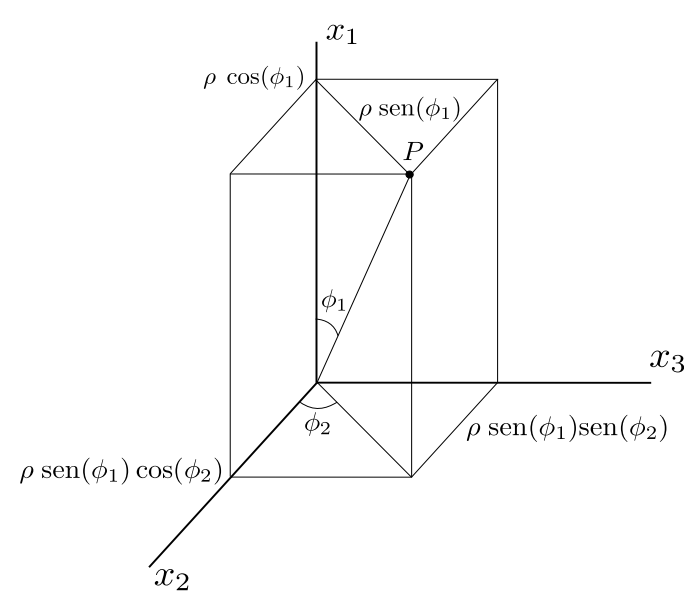

Figura 3.1: Coordenadas esféricas em $d=3$.

No aberto $U=(0, \infty) \times(0, \pi) \times(0, \pi), \times \cdots, \times(0,2 \pi)$, a função

$$
\begin{aligned}
\psi_{d}: U & \rightarrow \psi_{d}(U) \\
\left(\rho, \phi_{1}, \cdots, \phi_{d-1}\right) & \mapsto\left(x_{1}, x_{2}, \cdots, x_{d}\right)
\end{aligned}
$$

é um difeomorfismo (de fato, $\rho=\|x\|$ e os ângulos são obtidos sequencialmente). Os pontos de $\mathbb{R}^{d}$ fora de $\psi(U)$ têm medida zero, e não são relevantes para o cálculo das integrais.

Sejam $X \subset U$ e $\psi_{d}(X)$ mensuráveis. Mudando de variáveis, temos

$$
\lambda\left(\psi_{d}(X)\right)=\int_{\psi_{d}(X)} d x=\int_{X}\left|\operatorname{det} D \psi_{d}\left(\left(\rho, \phi_{1}, \cdots, \phi_{d-1}\right)\right)\right| d \rho d \phi_{1} \cdots d \phi_{d-1} .
$$


Proposição 3.3.1 Para $d \geq 3$ e para $\left(\rho, \phi_{1}, \cdots, \phi_{d-1}\right) \in U$ $\operatorname{det} D \psi_{d}\left(\left(\rho, \phi_{1}, \cdots, \phi_{d-1}\right)\right)=\rho^{d-1} \operatorname{sen}^{d-2}\left(\phi_{1}\right) \operatorname{sen}^{d-3}\left(\phi_{2}\right) \cdots \operatorname{sen}\left(\phi_{d-2}\right)>0$.

O caso $d=2$ é a troca de variáveis habitual para coordenadas polares: a fórmula vale com uma interpretação adequada.

Demonstração: A prova é uma indução em $d$. Escrevemos $c_{i}=\cos \left(\phi_{i}\right)$, $s_{i}=\operatorname{sen}\left(\phi_{i}\right)$ e $\operatorname{det} D \psi_{d}=\operatorname{det} D \psi_{d}\left(\rho, \phi_{1}, \cdots, \phi_{d-1}\right)$. Começamos mostrando o caso $d=3$ :

$$
\begin{aligned}
\operatorname{det} D \psi_{3} & =\operatorname{det}\left|\begin{array}{ccc}
c_{1} & -\rho s_{1} & 0 \\
s_{1} c_{2} & \rho c_{1} c_{2} & -\rho s_{1} s_{2} \\
s_{1} s_{2} & \rho c_{1} s_{2} & \rho s_{1} c_{2}
\end{array}\right| \\
& =\rho s_{1} c_{2} \operatorname{det}\left|\begin{array}{cc}
c_{1} & -\rho s_{1} \\
s_{1} c_{2} & \rho c_{1} c_{2}
\end{array}\right|+\rho s_{1} s_{2} \operatorname{det}\left|\begin{array}{cc}
c_{1} & -\rho s_{1} \\
s_{1} s_{2} & \rho c_{1} s_{2}
\end{array}\right| \\
& =\rho c_{1} c_{2}\left[\rho c_{1}^{2} c_{2}+\rho s_{1}^{2} c_{2}\right]+\rho s_{1} s_{2}\left[c_{1}^{2} \rho s_{2}+\rho s_{1}^{2} s_{2}\right] \\
& =\rho^{2} s_{1} c^{2}+\rho^{2} s_{1} s_{2}^{2}=\rho^{2} \operatorname{sen}\left(\phi_{1}\right) .
\end{aligned}
$$

Note que as duas matrizes $2 \times 2$ acima são obtidas multiplicando a segunda linha de $D \psi_{2}$ por $c_{2}$ e por $s_{2}$ respectivamente: a indução explora esse padrão.

A hípótese de indução para $d=k$ afirma que

$$
\begin{gathered}
\operatorname{det} D \psi_{k}=\operatorname{det}\left|\begin{array}{cccc}
c_{1} & -\rho s_{1} & \cdots & 0 \\
s_{1} c_{2} & \rho c_{1} c_{2} & \cdots & 0 \\
s_{1} s_{2} c_{3} & \rho c_{1} s_{2} c_{3} & \cdots & 0 \\
\vdots & \vdots & \vdots & \vdots \\
s_{1} \cdots c_{k-1} & \rho c_{1} \cdots c_{k-1} & \cdots & -\rho s_{1} \cdots s_{k-1} \\
s_{1} \cdots s_{k-1} & \rho c_{1} \cdots s_{k-1} & \cdots & \rho s_{1} \cdots c_{k-1}
\end{array}\right| \\
=\rho^{k-1} \operatorname{sen}^{k-2}\left(\phi_{1}\right) \operatorname{sen}^{k-3}\left(\phi_{2}\right) \cdots \operatorname{sen}\left(\phi_{k-2}\right) .
\end{gathered}
$$

Para $d=k+1$, temos

$$
\operatorname{det} D \psi_{k+1}=\operatorname{det}\left|\begin{array}{c|c}
E_{1} D \psi_{k} & \alpha e_{k} \\
\hline s_{k} e_{k}^{\top} D \psi_{k} & \beta
\end{array}\right|
$$


onde $e_{k}$ é o $k$-ésimo vetor canônico de $\mathbb{R}^{k}, \alpha=-\rho s_{1} \cdots s_{k-1} s_{k}, E_{1}=$ $\operatorname{diag}\left(1, \cdots, 1, c_{k}\right)$ e $\beta=\rho s_{1} \cdots s_{k-1} c_{k}$. Assim, concluímos a indução

$$
\begin{aligned}
\operatorname{det} D \psi_{k+1} & =\beta \operatorname{det}\left(E_{1} D \psi_{k}\right)-\alpha s_{k} \operatorname{det}\left(D \psi_{k}\right) \\
& =\rho s_{1} \cdots s_{k-1} c_{k} c_{k} \operatorname{det} D \psi_{k}+\rho s_{1} \cdots s_{k-1} s_{k} s_{k} \operatorname{det} D \psi_{k} \\
& =\rho s_{1} \cdots s_{k-1} \operatorname{det} D \psi_{k} \\
& =\rho^{k} \operatorname{sen}^{k-1}\left(\phi_{1}\right) \operatorname{sen}^{k-2}\left(\phi_{2}\right) \cdots \operatorname{sen}^{2}\left(\phi_{k-2}\right) \operatorname{sen}\left(\phi_{k-1}\right) .
\end{aligned}
$$

Como $\rho \in(0, \infty), \phi_{i} \in(0, \pi)$ para $i=1, \cdots, d-2$, então

$$
\operatorname{det} D \psi_{d}\left(\left(\rho, \phi_{1}, \cdots, \phi_{d-1}\right)\right)=\rho^{d-1} \operatorname{sen}^{d-2}\left(\phi_{1}\right) \operatorname{sen}^{d-3}\left(\phi_{2}\right) \cdots \operatorname{sen}\left(\phi_{d-2}\right)>0
$$

Assim,

$$
\lambda\left(\psi_{d}(X)\right)=\int_{X} \rho^{d-1} \operatorname{sen}^{d-2}\left(\phi_{1}\right) \operatorname{sen}^{d-3}\left(\phi_{2}\right) \cdots \operatorname{sen}\left(\phi_{d-2}\right) d \phi_{1} \cdots d \phi_{d-1} .
$$

Lembramos ao leitor que $\lambda=m \times \eta$, onde $m$ é uma medida em $(0, \infty)$ definida por $m(C)=\int_{C} \rho^{d-1} d \rho$, para todo boreliano $C \subset(0, \infty)$. Logo, para um boreliano $A \in S^{d-1}$,

$$
\eta(A)=\int_{\psi_{d}^{-1}(A)} \operatorname{sen}^{d-2}\left(\phi_{1}\right) \operatorname{sen}^{d-3}\left(\phi_{2}\right) \cdots \operatorname{sen}\left(\phi_{d-2}\right) d \phi_{1} \cdots d \phi_{d-1} .
$$

\section{4}

\section{Concentração em faixas equatoriais}

Vamos provar a concentração da esfera em faixas equatoriais. Para isso escolhemos um equador padrão, faixas equatoriais de espessura fixa e calotas. Nosso trabalho se resumirá a estimar áreas de calotas esféricas.

O equador padrão (ou simplesmente equador) em $S^{d-1}$ é o conjunto

$$
\begin{aligned}
E^{d-1} & =\left\{\left(x_{1}, \cdots, x_{d}\right) \in \mathbb{R}^{d}: x_{1}=0\right\} \\
& =\psi_{d}\left(\left\{\left(\rho, \phi_{1}, \cdots, \phi_{d-1}\right): \rho=1, \phi_{1}=\pi / 2\right\}\right) .
\end{aligned}
$$

Uma faixa equatorial $E_{\epsilon}^{d-1} \subset S^{d-1}$ de espessura $2 \epsilon \operatorname{com} \epsilon \in(0,1)$ é

$$
\begin{aligned}
E_{\epsilon}^{d-1} & =\left\{\left(x_{1}, \cdots, x_{d}\right) \in \mathbb{R}^{d}:-\epsilon<x_{1}<\epsilon\right\} \\
& =\psi_{d}\left(\left\{\left(\rho, \phi_{1}, \cdots, \phi_{d-1}\right): \rho=1, \pi / 2-\theta<\phi_{1}<\pi / 2+\theta\right\}\right)
\end{aligned}
$$

onde $\theta=\operatorname{arcsen}(\epsilon)$. 
A medida de probabilidade uniforme para os conjuntos borelianos da esfera é definida por normalização

$$
\sigma(A)=\frac{\eta(A)}{\eta\left(S^{d-1}\right)}
$$

Novamente, a invariância da medida de Lebesgue implica que $\sigma$ é invariante pela ação do grupo ortogonal $\mathcal{O}(d)^{2}$. A concentração da medida uniforme da esfera em faixas equatoriais de espessura $2 \epsilon$ se resume ao limite

$$
\lim _{d \rightarrow \infty} \sigma\left(S^{d-1} \backslash E_{\epsilon}^{d-1}\right)=0
$$

Claramente, $S^{d-1} \backslash E_{\epsilon}^{d-1}=\left\{\left(x_{1}, \cdots, x_{d}\right) \in \mathbb{R}^{d}: \epsilon<\left|x_{1}\right|<1\right\}$ é união de duas calotas esférica: uma calota é o subconjunto conexo da esfera acima ou abaixo de um plano $x_{1}=c$ com $-1<c<1$. Mais especificamente

$$
C_{N}^{d-1}(c)=\left\{x \in S^{d-1}: c<x_{1}<1\right\} ; \quad C_{S}^{d-1}(c)=\left\{x \in S^{d-1}:-1<x_{1}<c\right\} .
$$

Logo

$$
\sigma\left(S^{d-1} \backslash E_{\epsilon}^{d-1}\right)=\sigma\left(C_{N}^{d-1}(\epsilon)\right)+\sigma\left(C_{S}^{d-1}(-\epsilon)\right)=2 \sigma\left(C_{N}^{d-1}(\epsilon)\right)
$$

Vamos ver que a medida de calotas esféricas $C_{N}^{d}(\epsilon)$ de dimensão $d$ vai a zero quando $d \rightarrow \infty$. Em coordenadas esféricas,

$$
C_{N}^{d-1}(\epsilon)=\psi_{d}\left\{\left(\rho, \phi_{1} \cdot \cdots, \phi_{d-1}\right): \rho=1,0<\phi<\pi / 2-\theta\right\}
$$

Precisamos de uma estimativa.

Proposição 3.4.1 Para $0 \leq \alpha \leq \frac{\pi}{2}$, vale $\cos ^{d}(x) \leq \cos (x) \leq \exp \left(-x^{2} / 2\right)$.

Demonstração: $\mathrm{A} \operatorname{cota} \cos ^{d}(x) \leq \cos (x)$ é obvia. A fim de mostrar a outra, definimos

$$
f(x)=\ln (\cos (x))+\frac{x^{2}}{2}, \quad x \in[0, \pi / 2),
$$

cuja derivada é $f^{\prime}(x)=-\tan (x)+x$. Como $x<\tan (x)$ para $x \in[0, \pi / 2)$, concluímos que $f^{\prime}(x)<0$ no intervalo [0, $\left.\pi / 2\right)$. Logo, $f(x)<f(0) \forall x \in[0, \pi / 2)$, ou seja, $\ln (\cos (x))<\frac{-x^{2}}{2}$. O resultado segue de tomar a exponencial em ambos os lados.

\footnotetext{
${ }^{2} \mathrm{Na}$ seção 5.2 provamos que $\sigma$ é o push-forward da medida de Haar em $\mathcal{O}(d)$ e é a única medida invariante pelo grupo ortogonal $\mathcal{O}(d)$.
} 


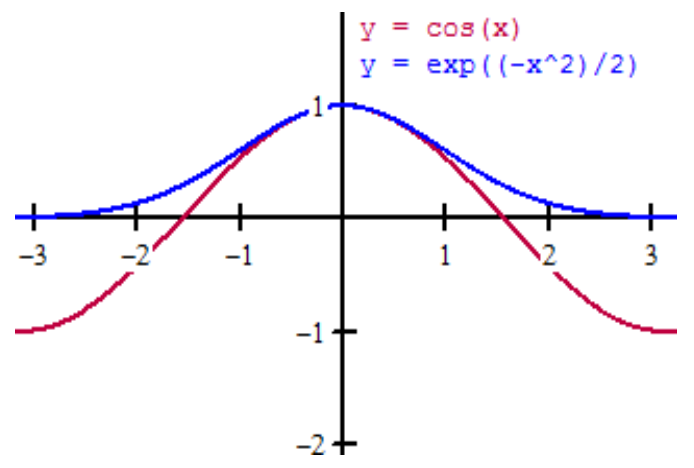

Figura 3.2: O gráfico representa a desigualdade $\cos (x) \leq \exp \left(-x^{2} / 2\right)$.

Vamos calcular a área da calota $C=C_{N}^{d+1}(\epsilon) \subset S^{d+1} \subset \mathbb{R}^{d+2}$

$$
\begin{aligned}
\eta(C) & =\int_{0}^{2 \pi} \cdots \int_{0}^{\pi} \int_{0}^{\pi / 2-\theta} \operatorname{sen}^{d}\left(\phi_{1}\right) \operatorname{sen}^{d-1}\left(\phi_{2}\right) \cdots \operatorname{sen}\left(\phi_{d}\right) d \phi_{1} \cdots d \phi_{d+1} \\
& =\int_{0}^{2 \pi} \cdots \int_{0}^{\pi} \operatorname{sen}^{d-1}\left(\phi_{2}\right) \cdots \operatorname{sen}\left(\phi_{d}\right) d \phi_{2} \cdots d \phi_{d+1} \int_{0}^{\pi / 2-\theta} \operatorname{sen}^{d}\left(\phi_{1}\right) d \phi_{1} \\
& =\eta\left(S^{d}\right) \int_{0}^{\pi / 2-\theta} \operatorname{sen}^{d}\left(\phi_{1}\right) d \phi_{1} .
\end{aligned}
$$

Como $\operatorname{sen}\left(\phi_{1}\right)=\cos \left(\phi_{1}-\frac{\pi}{2}\right)$, fazendo $\phi=\phi_{1}-\frac{\pi}{2}$ e $\frac{\Gamma\left(\frac{d+2}{2}\right)}{\Gamma\left(\frac{d+1}{2}\right) \sqrt{\pi}}=\alpha$, temos

$$
\begin{aligned}
\sigma(C) & =\frac{\eta(C)}{\eta\left(S^{d+1}\right)}=\frac{\eta\left(S^{d}\right)}{\eta\left(S^{d+1}\right)} \int_{0}^{\frac{\pi}{2}-\theta} \operatorname{sen}^{d}\left(\phi_{1}\right) d \phi_{1} \\
& =\alpha \int_{0}^{\frac{\pi}{2}-\theta} \operatorname{sen}^{d}\left(\phi_{1}\right) d \phi_{1}=\alpha \int_{\theta}^{\frac{\pi}{2}} \cos ^{d}(\phi) d \phi
\end{aligned}
$$

Usando $\phi=\frac{\psi}{\sqrt{d}}$ e aplicando a Proposição 3.4.1, temos

$$
\sigma(C) \leq \frac{\alpha}{\sqrt{d}} \int_{\theta \sqrt{d}}^{\frac{\pi}{2} \sqrt{d}} \mathrm{e}^{\frac{-\psi^{2}}{2}} d \psi
$$

Agora faça $\omega=\psi-\theta \sqrt{d}$

$$
\begin{aligned}
\sigma(C) & \leq \frac{\alpha}{\sqrt{d}} \mathrm{e}^{\frac{-\theta^{2} d}{2}} \int_{0}^{\sqrt{d}\left(\frac{\pi}{2}-\theta\right)} \mathrm{e}^{\frac{-\omega^{2}}{2}} d \omega \\
& \leq \frac{\alpha}{\sqrt{d}} \mathrm{e}^{\frac{-\theta^{2} d}{2}} \int_{0}^{\infty} \mathrm{e}^{\frac{-\omega^{2}}{2}} d \omega=\frac{\alpha}{\sqrt{d}} \frac{\sqrt{2 \pi}}{2} \mathrm{e}^{\frac{-\theta^{2} d}{2}} \\
& =\frac{\Gamma\left(\frac{d+2}{2}\right)}{\Gamma\left(\frac{d+1}{2}\right) \sqrt{2 d}} \exp \left(\frac{-\theta^{2} d}{2}\right)
\end{aligned}
$$


Fazendo $s_{d}=\frac{\Gamma\left(\frac{d+2}{2}\right)}{\Gamma\left(\frac{d+1}{2}\right) \sqrt{2 d}}$ e usando a equação $\Gamma(z+1)=z \Gamma(z)$, temos

$$
\begin{aligned}
s_{d+2} & =\frac{\Gamma\left(1+\frac{d+2}{2}\right)}{\Gamma\left(1+\frac{d+1}{2}\right)} \frac{1}{\sqrt{2(d+2)}}=\frac{\frac{d+2}{2} \Gamma\left(\frac{d+2}{2}\right)}{\frac{d+1}{2} \Gamma\left(\frac{d+1}{2}\right)} \frac{1}{\sqrt{2(d+2)}} \\
& =\left(\frac{\Gamma\left(\frac{d+2}{2}\right)}{\Gamma\left(\frac{d+1}{2}\right) \sqrt{2 d}}\right) \frac{d+2}{d+1} \frac{\sqrt{2 d}}{\sqrt{2(d+2)}}=s_{d} \frac{d+2}{d+1} \frac{\sqrt{2 d}}{\sqrt{2(d+2)}} \leq s_{d} .
\end{aligned}
$$

Logo, calculando $\max \left\{s_{1}, s_{2}\right\}=\frac{2}{\sqrt{\pi}}$, temos $s_{d} \leq \frac{2}{\sqrt{\pi}}, \forall d \in \mathbb{N}$. Portanto,

$$
\sigma\left(S^{d-1} \backslash E_{\epsilon}^{d-1}\right)=2 \sigma(C) \leq \frac{4}{\sqrt{\pi}} \exp \left(\frac{-\theta^{2} d}{2}\right)
$$

Fazendo $d \rightarrow \infty$, provamos a concentração da esfera

$$
\lim _{d \rightarrow \infty} \sigma\left(S^{d-1} \backslash E_{\epsilon}^{d-1}\right)=0
$$




\section{4}

\section{Análise em Grassmannianas}

O teorema de Johnson-Lindenstrauss emprega as projeções de $\mathbb{R}^{d}$ sobre subespaços vetoriais de dimensão $k$. O conjunto $G_{k}\left(\mathbb{R}^{d}\right)$ dos subespaços de dimensão $k$ em $\mathbb{R}^{d}$, chamado de Grassmanniana, é o objeto central do capítulo.

Apresentamos $G_{k}\left(\mathbb{R}^{d}\right)$ como um espaço métrico compacto, exibindo-o depois como subvariedade do espaço das matrizes simétricas, apresentando cartas locais e um atlas diferenciável.

Neste capítulo, se $E \in G_{k}\left(\mathbb{R}^{d}\right)$ então $P_{E}$ pode representar a projeção ortogonal sobre $E$ ou a matriz de $P_{E}$ na base canônica.

Seja

$$
\mathcal{G}(d, k):=\left\{P \in \mathcal{M}(d, \mathbb{R}): P^{2}=P, P^{*}=P^{T}=P, \operatorname{tr}(P)=k\right\},
$$

onde tr é função traço. Pelo teorema espectral, toda matriz simétrica $\left(P^{*}=P\right)$ e idempotente $\left(P^{2}=P\right)$ de posto $k$ se decompõe como

$$
P=Q \Lambda Q^{*}
$$

onde $Q \in \mathcal{O}(d)$ e $\Lambda$ é a matriz diagonal cujas $k$ primeiras entradas diagonais são iguais a 1, e as outras são nulas.

Como $\operatorname{tr}(A B)=\operatorname{tr}(B A)$, o traço é invariante por conjugação. Então uma matriz $P$ idempotente e simétrica tem $\operatorname{posto}(P)=k$ se, e somente se, $\operatorname{tr}(P)=k$. Assim,

$$
\mathcal{G}(d, k)=\left\{P \in \mathcal{M}(d, \mathbb{R}): P^{2}=P, P^{*}=P, \operatorname{posto}(P)=k\right\} .
$$

Proposição 4.0.2 A função $h: G_{k}\left(\mathbb{R}^{d}\right) \rightarrow \mathcal{G}(d, k), E \mapsto P_{E}$, é uma bijeção. $O$ conjunto $\mathcal{G}(d, k)$ é um espaço métrico compacto.

Assim, a identificação induz em $G_{k}\left(\mathbb{R}^{d}\right)$ uma estrutura de espaço métrico compacto. Na verdade, todas as propriedades de interesse da Grassmanniana serão estudadas em $\mathcal{G}(d, k)$, e meramente transferidas para a descrição em termos de subespaços. 
Demonstração: Fixada a base canônica em $\mathbb{R}^{d}$, existe um isomorfismo entre $\mathcal{M}(d, \mathbb{R})$ e $\mathcal{L}\left(\mathbb{R}^{d}\right)$, o conjunto de todos os operadores lineares $T: \mathbb{R}^{d} \rightarrow \mathbb{R}^{d}$. Lembramos que toda projeção ortogonal $P: \mathbb{R}^{d} \rightarrow \mathbb{R}^{d}$ sobre um subespaço $E \subset \mathbb{R}^{d}$ é autoadjunta, idempotente e satisfaz posto $(P)=\operatorname{dim} E$. Assim, para cada $E \in G_{k}\left(\mathbb{R}^{d}\right)$ designamos $P_{E}: \mathbb{R}^{d} \rightarrow \mathbb{R}^{d}$, a matriz(única) da projeção ortogonal sobre $E$, na base canônica. Logo, a função $h$, além de estar bem definida, é injetiva. Agora provaremos a sobrejetividade. Seja $P \in \mathcal{G}(d, k)$. Pela bijeção entre $\mathcal{M}(d, \mathbb{R})$ e $\mathcal{L}\left(\mathbb{R}^{d}\right)$, tomamos o operador $P_{E}: \mathbb{R}^{d} \rightarrow \mathbb{R}^{d}$ correspondente à matriz $P$ que claramente é idempotente e autoadjunto. Além disso, $\operatorname{Im}(P)$ tem dimensão $k$. Ou seja, $P$ é uma projeção ortogonal sobre $E:=\operatorname{Im} P \in G_{k}\left(\mathbb{R}^{d}\right)$. Logo $h(E)=P$.

A norma de Frobenius de $A \in \mathcal{M}(d, \mathbb{R})$ é dada por $\|A\|^{2}=\operatorname{tr}\left(A^{*} A\right)$. Para $P \in \mathcal{G}(d, k)$

$$
\|P\|^{2}=\operatorname{tr}\left(P_{E}^{*} P_{E}\right)=\operatorname{tr}\left(P_{E}^{2}\right)=\operatorname{tr}\left(P_{E}\right)=k,
$$

mostrando que $\mathcal{G}(d, k)$ é limitado.

Para ver que $\mathcal{G}(d, k)$ é fechado, note que ele é a interseção de níveis de várias funções contínuas (os níveis zero de $\left(P^{2}-P\right)$, de $\left(P^{*}-P\right)$ e de $(\operatorname{tr} P-k)$ ).

Portanto, $\mathcal{G}(d, k)$ é fechado e mais, compacto.

\section{1}

\section{$G_{k}\left(\mathbb{R}^{d}\right)$ como subvariedade de $\mathcal{S}\left(\mathbb{R}^{d}\right)$}

Considere a ação por conjugação do grupo $\mathcal{O}(d)$ sobre o conjunto das matrizes reais simétricas $\mathcal{S}\left(\mathbb{R}^{d}\right)$

$$
\begin{aligned}
\phi: \mathcal{O}(d) \times \mathcal{S}\left(\mathbb{R}^{d}\right) & \rightarrow \mathcal{S}\left(\mathbb{R}^{d}\right) \\
(Q, S) & \mapsto Q S Q^{*}
\end{aligned}
$$

Se duas matrizes simétricas $S$ e $\Lambda$ têm os mesmos autovalores então, pelo teorema espectral, as orbitas da ação por conjugação passando por $S$ e $\Lambda$ são iguais. Para a matriz $\Lambda$ descrita em (4-1), as matrizes da órbita são todas idempotentes, simétricas e do mesmo traço. Assim a órbita de $\Lambda$ coincide com $\mathcal{G}(d, k)$.

Usaremos a Proposição 2.0.1, anunciada no capítulo 2, para provar que $\mathcal{G}(d, k)=\mathcal{O}(d) \Lambda$ é uma subvariedade fechada de $\mathcal{S}\left(\mathbb{R}^{d}\right):$

Proposição 1 Seja $\phi: G \times M \rightarrow M$ uma ação própria. Então toda órbita $G x$ é uma subvariedade fechada de $M$. 
Lembramos ao leitor que uma ação $\phi$ é própria se $\tilde{\phi}: G \times M \rightarrow M \times M$, $(g, x) \mapsto(x, \phi(g, x))$, é uma aplicação própria, isto é, se a inversa de um compacto é compacto.

Corolário 4.1 $\mathcal{G}(d, k)$ é uma subvariedade fechada de $\mathcal{S}\left(\mathbb{R}^{d}\right)$.

Demonstração: Neste caso, $\tilde{\phi}: \mathcal{O}(d) \times \mathcal{S}\left(\mathbb{R}^{d}\right) \rightarrow \mathcal{S}\left(\mathbb{R}^{d}\right) \times \mathcal{S}\left(\mathbb{R}^{d}\right)$ é dada por $\tilde{\phi}(Q, S)=(S, \phi(Q, S))=\left(S, Q S Q^{*}\right)$. Tome $K=K_{1} \times K_{2} \subset \mathcal{S}\left(\mathbb{R}^{d}\right) \times \mathcal{S}\left(\mathbb{R}^{d}\right)$ com $K_{1}$ e $K_{2}$ compactos. Provaremos que

$$
\tilde{K}=(\tilde{\phi})^{-1}(K)=\left\{(Q, S) \in \mathcal{O}(d) \times \mathcal{S}\left(\mathbb{R}^{d}\right): S \in K_{1}, \phi(Q, S) \in K_{2}\right\}
$$

é um compacto. É claro que $\tilde{K}$ é fechado, pois $\tilde{\phi}$ é contínua e $K$ é compacto. Logo, resta mostrar que $\tilde{K}$ é limitado. Ora, $\tilde{K} \subset \mathcal{O}(d) \times K_{1}$ onde $\mathcal{O}(d)$ e $K_{1}$, ambos limitados. Logo, $\tilde{K}$ é limitado.

\section{2}

\section{A função posto}

Seja uma $A=\left(a_{i j}\right)$ uma matriz de dimensão $m \times n$. Nesta seção, $A_{i j}$ é a submatriz de A de dimensão $i \times j$ com entradas na interseção das $i$ primeiras linhas com as $j$ primeiras colunas de $A$. O exemplo é esclarecedor.

$$
A=\left(\begin{array}{ll|ll}
3 & 1 & 1 & 0 \\
2 & 1 & 2 & 8 \\
2 & 2 & 4 & 7 \\
6 & 5 & 1 & 0 \\
3 & 1 & 4 & 2
\end{array}\right)_{5 \times 4} \quad A_{32}=\left(\begin{array}{ll}
3 & 1 \\
2 & 1 \\
2 & 2
\end{array}\right)_{3 \times 2}
$$

Seja $A=\left(a_{i j}\right)$ uma matriz $m \times n$. Definimos a função posto $\mathcal{P}_{A}$

$$
\begin{aligned}
\mathcal{P}_{A}:\{1,2, \cdots, m\} \times\{1,2, \cdots, n\} & \rightarrow \mathbb{N} \cup\{0\} \\
(i, j) & \mapsto \operatorname{posto}\left(A_{i j}\right)
\end{aligned}
$$

Damos uma visualização matricial à função $\mathcal{P}_{A}$. A matriz posto de $A$ é a matriz $\mathrm{P}_{A}=\left(p_{i j}\right)_{m \times n}$ onde $p_{i j}=\mathcal{P}_{A}(i, j)$.

\section{Exemplo:}

$$
A=\left(\begin{array}{lll}
0 & 0 & 3 \\
2 & 1 & 2 \\
2 & 1 & 4 \\
6 & 5 & 1
\end{array}\right)_{4 \times 3} \quad \mathrm{P}_{A}=\left(\begin{array}{lll}
0 & 0 & 1 \\
1 & 1 & 2 \\
1 & 1 & 2 \\
1 & 2 & 3
\end{array}\right)_{4 \times 3}
$$


A configuração local em $i j$ da matriz $\mathrm{P}_{A}$ é a submatriz

$$
\begin{array}{c|c}
p_{(i-1)(j-1)} & p_{(i-1) j} \\
\hline p_{i(j-1)} & p_{i j}
\end{array} .
$$

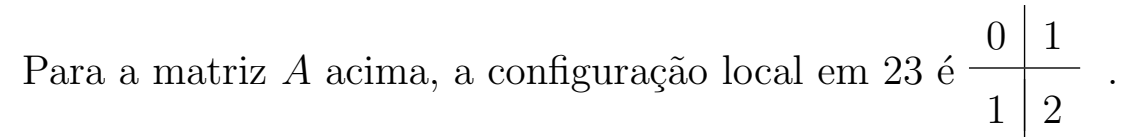

Observamos duas propriedades simples da função posto: na primeira linha ou coluna da matriz $A$ a função $\mathcal{P}_{A}$ toma valores iguais a 0 ou 1 e quando a matriz aumenta uma linha ou uma coluna, o posto pode aumentar em no máximo 1. Ou seja, $\mathcal{P}_{A}$ é monótona, no seguinte sentido

$$
p_{i j} \leq p_{(i+1) j} \leq p_{i j}+1, \quad p_{i j} \leq p_{i(j+1)} \leq p_{i j}+1
$$

Os seguintes diagramas mostram as configurações locais possíveis:

$$
\begin{aligned}
& \frac{p \mid p}{p \mid p}, \frac{p \mid p}{p \mid p+1}, \frac{p \mid p+1}{p \mid p+1}, \\
& \begin{array}{c|c}
p & p \\
p+1 & p+1
\end{array}, \frac{p}{p+1 \mid p+1}, \frac{p+1}{p+1} \mid p+2
\end{aligned}
$$

Porém a proposição seguinte descarta a quinta configuração.

Proposição 4.2.1 Seja $A=\left(a_{i j}\right)_{m \times n}$ uma matriz e $\mathrm{P}_{A}=\left(p_{i j}\right)_{m \times n}$ sua matriz posto. Se $p_{i j}=p, p_{i(j+1)}=p+1$ e $p_{(i+1) j}=p+1$, então $p_{(i+1)(j+1)}=p+2$.

Demonstração: Ao longo desta prova, para cada $v \in \mathbb{R}^{i+1}, v^{\prime} \in \mathbb{R}^{i}$ denotará o vetor obtido truncando a última coordenada de $v$. A demonstração é por absurdo. Como

$$
\mathrm{P}_{A_{(i+1)(j+1)}}=\left(\begin{array}{r|r} 
& \\
p & p+1 \\
\hline p+1 & p+1
\end{array}\right)=\left(\begin{array}{rr}
\mathrm{P}_{A_{(i+1) j}} & \\
& \\
& p+1
\end{array}\right),
$$

existem $p+1$ colunas LI em $A_{(i+1) j}$.

Sejam $\left\{v_{1}, v_{2}, \cdots, v_{p}, v_{p+1}\right\}$ tais vetores em $\mathbb{R}^{i+1}$ e $\left\{v_{1}^{\prime}, v_{2}^{\prime}, \cdots, v_{p}^{\prime}, v_{p+1}^{\prime}\right\}$ seus truncamentos em $\mathbb{R}^{i}$.

Fato 1. Exatamente $p$ vetores de $\left\{v_{1}^{\prime}, v_{2}^{\prime}, \cdots, v_{p}^{\prime}, v_{p+1}^{\prime}\right\}$ são $L I$.

Demonstração: Seja $k<p$ e suponha que no máximo $k$ vetores de $\left\{v_{1}^{\prime}, v_{2}^{\prime}, \cdots, v_{p}^{\prime}, v_{p+1}^{\prime}\right\}$ sejam LI. Sem perda de generalidade sejam 
$\left\{v_{1}^{\prime}, v_{2}^{\prime}, \cdots, v_{k}^{\prime}\right\}$ tais vetores. Temos então que

$$
\operatorname{dim}\left(\operatorname{span}\left\{v_{1}^{\prime}, v_{2}^{\prime}, \cdots, v_{p}^{\prime}, v_{p+1}^{\prime}\right\}\right)=k .
$$

Logo

$$
\operatorname{dim}\left(\operatorname{span}\left\{v_{1}, v_{2}, \cdots, v_{p}, v_{p+1}\right\}\right) \leq k+1 \leq p,
$$

um absurdo pois $\left\{v_{1}, v_{2}, \cdots, v_{p}, v_{p+1}\right\}$ é um conjunto LI. Por outro lado, $\left\{v_{1}^{\prime}, v_{2}^{\prime}, \cdots, v_{p}^{\prime}, v_{p+1}^{\prime}\right\}$ não pode ser LI, pois $\left\{v_{1}^{\prime}, v_{2}^{\prime}, \cdots, v_{p}^{\prime}, v_{p+1}^{\prime}\right\}$ são colunas da submatriz $A_{i j}$ que tem posto $p$. Assim concluímos que exatamente $p$ vetores de $\left\{v_{1}^{\prime}, v_{2}^{\prime}, \cdots, v_{p}^{\prime}, v_{p+1}^{\prime}\right\}$ são LI.

Sem perda, sejam $\left\{v_{1}^{\prime}, v_{2}^{\prime}, \cdots, v_{p}^{\prime}\right\}$ esses $p$ vetores. Então

$$
v_{p+1}^{\prime}=\beta_{1} v_{1}^{\prime}+\beta_{2} v_{2}^{\prime}, \cdots, \beta_{p} v_{p}^{\prime}
$$

Seja $w$ a última coluna da submatriz $A_{(i+1)(j+1)}$ e considere seu truncamento $w^{\prime}$, a última coluna de $A_{i(j+1)}$

$$
\mathrm{P}_{A_{(i+1)(j+1)}}=\left(\begin{array}{l|l} 
& \\
\mathrm{P}_{A_{i(j+1)}} & \mathrm{P}_{w^{\prime}} \\
& \mid p+1 \\
&
\end{array}\right) .
$$

Fato 2. $\left\{v_{1}^{\prime}, v_{2}^{\prime}, \cdots, v_{p}^{\prime}, w^{\prime}\right\}$ é um conjunto $L I$.

Demonstração: Por hipótese $p_{i(j+1)}=p+1$. Logo a submatriz $A_{i(j+1)}$ tem $p+1$ colunas LI, onde necessariamente, $w^{\prime}$ é uma dessas colunas. Caso contrário $A_{i j}$ teria $p+1$ colunas LI, contradizendo o fato da submatriz ter posto $p$. Tomamos então essas $p+1$ colunas LI como sendo $\left\{u_{1}, \cdots, u_{p}, w^{\prime}\right\}$. Ora, $\left\{u_{1}, \cdots, u_{p}\right\}$ e $\left\{v_{1}^{\prime}, v_{2}^{\prime}, \cdots, v_{p}^{\prime}\right\}$ são duas bases para o subespaço gerado pelas colunas de $A_{i j}$, onde $w^{\prime}$ não pertence a esse subespaço, pois $\left\{w^{\prime}, u_{1}, \cdots, u_{p}\right\}$ é um conjunto LI. Assim, $\left\{v_{1}^{\prime}, v_{2}^{\prime}, \cdots, v_{p}^{\prime}, w^{\prime}\right\}$ é LI.

Como $\left\{v_{1}, v_{2}, \cdots, v_{p}, v_{p+1}\right\}$ é LI em $\mathbb{R}^{i+1}$, temos, para $\alpha^{\prime} s$ adequados,

$$
w=\alpha_{1} v_{1}+\alpha_{2} v_{2}, \cdots, \alpha_{p} v_{p}+\alpha_{p+1} v_{p+1}
$$

$\mathrm{e}, \mathrm{em} \mathbb{R}^{i}$

$$
w^{\prime}=\alpha_{1} v_{1}^{\prime}+\alpha_{2} v_{2}^{\prime}, \cdots, \alpha_{p} v_{p}^{\prime}+\alpha_{p+1} v_{p+1}^{\prime}
$$


Substituindo (4-2) em (4-3),

$$
\begin{aligned}
w^{\prime} & =\alpha_{1} v_{1}^{\prime}+\alpha_{2} v_{2}^{\prime}, \cdots, \alpha_{p} v_{p}^{\prime}+\alpha_{p+1}\left(\beta_{1} v_{1}^{\prime}+\beta_{2} v_{2}^{\prime}, \cdots, \beta_{p} v_{p}^{\prime}\right) \\
& =\left(\alpha_{1}+\alpha_{p+1} \beta_{1}\right) v_{1}^{\prime}+\cdots+\left(\alpha_{p}+\alpha_{p+1} \beta_{p}\right) v_{p}^{\prime},
\end{aligned}
$$

um absurdo, pois $\left\{v_{1}^{\prime}, v_{2}^{\prime}, \cdots, v_{p}^{\prime}, w^{\prime}\right\}$ é LI, pelo fato anterior.

Exemplo: A existência de cada uma das configurações locais remanescentes

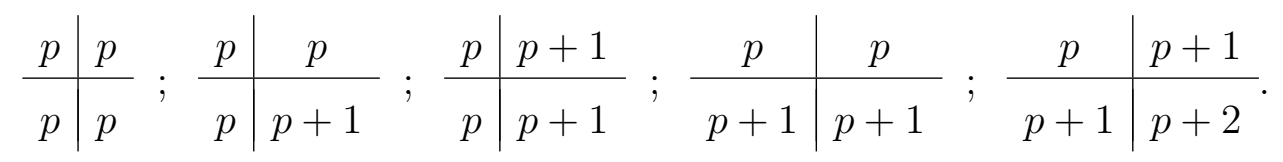

pode ser verificada a seguir

$$
A=\left(\begin{array}{cccccc}
0 & 0 & 1 & 9 & 0 & 1 \\
3 & 8 & 6 & 21 & 16 & \mathbf{7} \\
0 & 5 & \mathbf{2} & 7 & 10 & 53 \\
7 & 1 & 32 & 0 & 2 & 4 \\
0 & 0 & 2 & 18 & \mathbf{0} & 2 \\
2 & 3 & 7 & 8 & 6 & 1 \\
0 & \mathbf{3} & 84 & 2 & 6 & \mathbf{0}
\end{array}\right)_{7 \times 6} \quad \mathrm{P}_{A}=\left(\begin{array}{cccccc}
0 & 0 & 1 & 1 & \mathbf{1} & \mathbf{1} \\
1 & \mathbf{1} & \mathbf{2} & 2 & \mathbf{2} & \mathbf{2} \\
1 & \mathbf{2} & \mathbf{3} & 3 & 3 & 3 \\
1 & 2 & 3 & \mathbf{4} & \mathbf{4} & 4 \\
1 & 2 & 3 & \mathbf{4} & \mathbf{4} & 4 \\
\mathbf{1} & \mathbf{2} & 3 & 4 & \mathbf{4} & \mathbf{4} \\
\mathbf{1} & \mathbf{2} & 3 & 4 & \mathbf{4} & \mathbf{5}
\end{array}\right)_{7 \times 6}
$$

Para 66, 62, 55, 33, 26 as configurações locais são

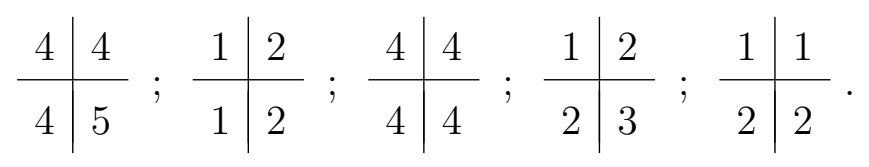

Vamos emoldurar $A$ com uma primeira linha e uma primeira coluna de zeros, como abaixo. As entradas da matriz aumentada $\tilde{A}=\left(\tilde{a}_{i j}\right)_{(m+1) \times(n+1)}$ são indexadas a partir de 0

$$
A=\left(\begin{array}{cccc}
a_{11} & a_{12} & \cdots & a_{1 n} \\
a_{21} & a_{22} & \cdots & a_{2 n} \\
\vdots & \vdots & & \vdots \\
a_{m 1} & a_{m 2} & \cdots & a_{m n}
\end{array}\right), \quad \tilde{A}=\left(\begin{array}{ccccc}
0 & 0 & 0 & \cdots & 0 \\
0 & a_{11} & a_{12} & \cdots & a_{1 n} \\
0 & a_{21} & a_{22} & \cdots & a_{2 n} \\
\vdots & \vdots & \vdots & & \vdots \\
0 & a_{m 1} & a_{m 2} & \cdots & a_{m n}
\end{array}\right)
$$

Seja $A=\left(a_{i j}\right)$ uma matriz $m \times n$ e $\tilde{A}=\left(\tilde{a}_{i j}\right)$ sua matriz aumentada. Vamos marcar algumas entradas de $A$ : as entradas $i j$ de $\tilde{A}$ cuja configuração local é dada por $\frac{p}{p} \mid$\begin{tabular}{c}
$p+1$ \\
\hline$p$
\end{tabular} .

A configuração de torres de $A$ é a matriz $\mathcal{T}(A)$ de zeros e uns, da 
mesma dimensão de $A$, onde os uns indicam as entradas marcadas em $A$. A palavra torre é empregada para sugerir que, como veremos no primeiro item da Proposição 4.2.2, não é possível ter duas entradas marcadas ou na mesma linha ou na mesma coluna, isto é, duas torres não se atacam.

Exemplo: Seja $A$ a matriz do exemplo anterior. A matriz aumentada $\tilde{A}$, a matriz posto de $\tilde{A}$ e a configuração de torres de $A$ são, respectivamente,

$$
\tilde{A}=\left(\begin{array}{ccccccc}
0 & 0 & 0 & 0 & 0 & 0 & 0 \\
0 & 0 & 0 & 1 & 9 & 0 & 1 \\
0 & 3 & 8 & 6 & 21 & 16 & 7 \\
0 & 0 & 5 & 2 & 7 & 10 & 53 \\
0 & 7 & 1 & 32 & 0 & 2 & 4 \\
0 & 0 & 0 & 2 & 18 & 0 & 2 \\
0 & 2 & 3 & 7 & 8 & 6 & 1 \\
0 & 0 & 3 & 84 & 2 & 6 & 0
\end{array}\right)_{8 \times 7} \quad \mathrm{P}_{\tilde{A}}=\left(\begin{array}{ccccccc}
0 & 0 & 0 & 0 & 0 & 0 & 0 \\
0 & 0 & 0 & \mathbf{1} & 1 & 1 & 1 \\
0 & \mathbf{1} & 1 & 2 & 2 & 2 & 2 \\
0 & 1 & \mathbf{2} & 3 & 3 & 3 & 3 \\
0 & 1 & 2 & 3 & 4 & 4 & 4 \\
0 & 1 & 2 & 3 & 4 & 4 & 4 \\
0 & 1 & 2 & 3 & 4 & 4 & 4 \\
0 & 1 & 2 & 3 & 4 & 4 & 5
\end{array}\right)_{8 \times 7}
$$

$$
\mathcal{T}(A)=\left(\begin{array}{llllll}
0 & 0 & \mathbf{1} & 0 & 0 & 0 \\
\mathbf{1} & 0 & 0 & 0 & 0 & 0 \\
0 & \mathbf{1} & 0 & 0 & 0 & 0 \\
0 & 0 & 0 & \mathbf{1} & 0 & 0 \\
0 & 0 & 0 & 0 & 0 & 0 \\
0 & 0 & 0 & 0 & 0 & 0 \\
0 & 0 & 0 & 0 & 0 & 1
\end{array}\right)_{7 \times 6}
$$

É claro que, se na primeira linha (ou coluna) de qualquer matriz não existem torres, então as entradas dessa linha(ou coluna) são todas iguais a 0 .

Lema 4.2 Seja $A=\left(a_{i j}\right)$ uma matriz $m \times n$. Suponha que na linha $r>1$ não existam torres. Então, em $\mathrm{P}_{A}=\left(p_{i j}\right)$ temos $p_{r j}=p_{(r-1) j}, \forall j=1,2, \cdots n$. Ou seja, a linha $r$ da matriz $\mathrm{P}_{A}$ é uma cópia da sua antecessora. O resultado é análogo para colunas.

Demonstração: Mostramos o resultado para linhas. Tome a primeira coluna $k \in\{1,2, \cdots, n\}$ com $p_{r k} \neq p_{(r-1) k}$. Se $p_{(r-1) k}=p$, então pela monotonicidade da função posto, a configuração local na entrada $r k$ é

$$
\begin{array}{c|c}
p_{(r-1)(k-1)} & p \\
\hline p_{r(k-1)} & \boldsymbol{p}+\mathbf{1}
\end{array}
$$


e os únicos valores possíveis para $p_{(r-1)(k-1)}$ e $p_{r(k-1)}$ são

$$
\begin{array}{c|c}
p & p \\
\hline p & \boldsymbol{p}+\mathbf{1}
\end{array}
$$

o que implicaria a existência de uma torre na linha $r$, um absurdo.

\section{Proposição 4.2.2 Valem as seguintes propriedades:}

1. As torres de uma matriz $A=\left(a_{i j}\right)_{m \times n}$ não se atacam. Ou seja, em cada linha ou coluna da matriz $A$, existe no máximo uma torre.

2. O número de torres de qualquer matriz $A=\left(a_{i j}\right)_{m \times n}$ é igual ao posto da mesma.

Demonstração: Provaremos só o caso das linhas, o outro é análogo. Suponha que na entrada $a_{r k}$ exista uma torre

$$
\begin{array}{c|c}
a_{(r-1)(k-1)} & a_{(r-1) k} \\
\hline a_{r(k-1)} & \boldsymbol{a}_{r k}
\end{array} \quad \begin{aligned}
& p \\
& p
\end{aligned}
$$

Dentro das possíveis configurações, existem só duas possibilidades ao prenecher a coluna $k+1$ nas linhas $r$ e $r-1$

$$
\begin{array}{c|c}
p & p \\
\hline p+1 & p+1
\end{array}, \quad \begin{array}{c|c}
p & p+1 \\
\hline p+1 & p+2
\end{array} .
$$

Repetindo este processo, concluímos que não pode haver outra torre na mesma linha $r$. Assim, provamos o primeiro item. A prova do segundo item será feita por indução na variável $m$ fixando a variável $n$. Para $m=1$ o resultado é bastante claro. Suponha que para toda matriz de dimensão $m \times n$ o número de torres é igual ao posto. Seja então uma matriz $A=\left(a_{i j}\right)_{(m+1) \times n}$. Nosso objetivo é provar que $A$ tem posto igual ao número de torres. Considere a matriz posto de $A$

$$
\mathrm{P}_{A}=\left(\begin{array}{cccc}
p_{11} & p_{12} & \cdots & p_{1 n} \\
p_{21} & p_{22} & \cdots & p_{2 n} \\
\vdots & \vdots & & \vdots \\
p_{m 1} & p_{m 2} & \cdots & p_{m n} \\
p_{(m+1) 1} & p_{(m+1) 2} & \cdots & p_{(m+1) n}
\end{array}\right) .
$$

Se retirarmos a última linha da matriz $A$ obtemos uma submatriz $A_{m n}$. Pela hipótese de indução o número de torres de $A_{m n}$ é igual ao seu 
posto. Assumimos $p_{(m+1) \times n} \neq 0$. Caso contrário o resultado é trivial. Assim, denotamos $p_{(m+1) \times n}=p+1$

$$
\mathrm{P}_{A}=\left(\begin{array}{c|c}
p_{m n} \text { torres } & \\
\cline { 2 - 2 } & p_{m n} \\
\hline & p+1
\end{array}\right)_{(m+1) \times n} .
$$

Pela monotonicidade da função posto, temos dois possíveis valores para $p_{m n}$. A primeira é $p_{m n}=p$

$$
\mathrm{P}_{A}=\left(\begin{array}{cc|}
p \text { torres } \\
\cline { 2 - 2 } & p \\
\hline & p+1
\end{array}\right)_{(m+1) \times n} .
$$

Neste caso, necessariamente existe uma torre na ultima linha, caso contrário, pelo Lema 4.2 teriamos $p_{m n}=p_{(m-1) n}$. Assim, o numero de torres da matriz $A$ é igual ao seu posto. Finalmente, a outra possibilidade é $p_{m n}=p+1$

$$
\mathrm{P}_{A}=\left(\begin{array}{lr}
p+1 \text { torres } \\
\cline { 2 - 2 } & p+1 \\
\hline & p+1
\end{array}\right)_{(m+1) \times n} .
$$

Neste caso, não pode existir uma torre na ultima linha $\mathrm{P}_{A}$. Se existe uma torre nessa linha, usamos o mesmo argumento usado na prova do primeiro item para concluir que $p_{(m+1) n}=p_{m n}+1$, um absurdo.

Corolário 4.3 Toda matriz A quadrada de dimensão $n$ é inversível se, e somente se, A tem n torres. Além disso, ao permutarmos duas linhas ou duas colunas de uma matriz qualquer, então o número de torres não se altera.

Demonstração: Este resultado é consequência imediata do segundo item da proposição anterior. Se uma matriz quadrada $A$ de dimensão $n$ é inversível, então $\operatorname{posto}(A)=n$. Assim, $A$ tem $n$ torres. Reciprocamente, se $A=\left(a_{i j}\right)$ é uma matriz quadrada de dimensão $n$ possuindo $n$ torres então $\operatorname{posto}(A)=n$, $\operatorname{logo} A$ é inversível. Ao permutarmos duas linhas ou colunas da matriz $A$ o seu posto não muda, logo o número de torres se mantém.

Proposição 4.2.3 Seja $Q$ uma matriz $d \times k$ com $d \geq k$. Se $Q$ possui $k$ torres, ou seja, uma torre em cada coluna, então existe uma matriz permutação $\Pi$ de 
modo que $\Pi Q$ tem a seguinte configuração de torres.

$$
\mathcal{T}(\Pi Q)=\left(\begin{array}{cccc}
\mathbf{1} & 0 & 0 & 0 \\
0 & \mathbf{1} & 0 & 0 \\
\vdots & \vdots & \ddots & \vdots \\
0 & 0 & 0 & \mathbf{1} \\
& & & \\
& & &
\end{array}\right)_{d \times k} .
$$

Demonstração: Para qualquer permutação, ou sequência de permutações, das linhas de $Q$, corresponde uma matriz permutação. Lembramos a notação de submatriz: $Q_{d j}$ representa a submatriz de $Q$ cujas colunas são exatamente as $j$ primeiras colunas de $Q$.

Por hipótese, na primeira coluna de $Q$ existe uma torre. Logo, nessa mesma coluna, existe uma entrada diferente de zero. Suponha que essa entrada não nula se encontre na linha $r \neq 1$ da matriz $Q$. Ao permutarmos a linha $r$ com a primeira linha de $Q$ obtemos uma matriz permutação $\Pi_{1}$ tal que $Q^{1}=\Pi_{1} Q=\left(q_{i j}^{1}\right)$ tem uma torre na entrada $q_{11}^{1}$. Além disso, $Q^{1}$ continua com $k$ torres e uma em cada coluna. Agora tomemos a submatriz $Q_{d 2}^{1}$. Sabemos que existem duas torres na submatriz $Q_{d 2}^{1}$ onde uma delas se encontra na posição $(1,1)$. Suponha que a segunda torre de $Q_{d 2}^{1}$ se encontra na posição $(r, 2)$. Ora, pelo segundo item do Proposição 4.2.2, a submatriz $Q_{d 2}^{1}$ tem posto 2, isto é, $Q_{d 2}^{1}$ tem duas linhas linearmente independentes. Pelo Lema 4.2, é fácil concluir que todas as linhas de $Q_{d 2}^{1}$ entre as duas torres são linearmente dependentes da primeira. Concluímos assim que as linhas 1 e $r$ da submatriz $Q_{d 2}^{1}$ são LI e ao permutarmos as linhas 2 e $r$, obtemos uma matriz permutação $\Pi_{2}$ tal que $Q^{2}=\Pi_{2} Q^{1}=\Pi_{2} \Pi_{1} Q=\left(q_{i j}^{2}\right)$ tem duas torres nas entradas $q_{11}^{2}$ e $q_{22}^{2}$. Além disso, $Q^{2}$ continua com $k$ torres e uma em cada coluna. Continuando este processo, obtemos uma matriz permutação $\Pi=\Pi_{k} \circ \Pi_{k-1} \circ \cdots \circ \Pi_{1}$ tal que $\Pi Q=Q^{k}=\left(q_{i j}^{k}\right)$ tem as $k$ torres em $q_{11}^{k}, q_{22}^{k}, \cdots, q_{k k}^{k}$.

\section{3}

\section{$G_{k}\left(\mathbb{R}^{d}\right)$ como variedade diferenciável}

Já provamos que $G_{k}\left(\mathbb{R}^{d}\right)$ é uma subvariedade de $\mathcal{S}\left(\mathbb{R}^{d}\right)$. Nesta seção exibiremos cartas.

Para $Q \in \mathcal{O}(d)$, defina $Q_{k}$ a matriz com as $k$ primeiras colunas de $Q$. Se $A$ é uma matriz quadrada de dimensão $k$, escrevemos $\mathcal{T}(A)=\mathbb{I}_{k}$, para indicar 
que $A$ tem as torres na diagonal, ou seja, a configuração de torres de $A$ é igual à configuração de torres da matriz identidade de dimensão $k$.

Se $E \in G_{k}\left(\mathbb{R}^{d}\right)$ e $P_{E}$ é a matriz da projeção ortogonal sobre $E$, lembramos ao leitor que pelo teorema espectral

$$
P_{E}=Q \Lambda Q^{*}
$$

onde $Q \in \mathcal{O}(d)$ e $\Lambda$ é a matriz diagonal cujas $k$ primeiras entradas diagonais são iguais a 1 , e as outras são nulas. As colunas de $Q_{k}$ formam uma base de $E$, $\operatorname{logo} \operatorname{posto}\left(Q_{k}\right)=k$. Portanto, $Q_{k}$ tem $k$ torres e, pela Proposição 4.2.3, existe uma matriz de permutação $\Pi$ tal que

$$
\Pi Q_{k}=\left[\frac{A}{B}\right], \quad \mathcal{T}(A)=\mathbb{I}_{k}
$$

Finalmente, pelo Corolário 4.3, $A$ é inversível. Feito este procedimento, determinamos as cartas locais.

Sejam $\mathcal{I}_{d}=\{1,2, \cdots, d\}$ e $S_{d}$ o grupo simétrico correspondente, cujos elementos são as permutações de $\mathcal{I}_{d}$. Para cada $\pi \in S_{d}$, denota-se $\Pi_{\pi}$ a matriz de permutação correspondente. Seja

$$
U_{\pi}=\left\{E \in G_{k}\left(\mathbb{R}^{d}\right): P_{E}=Q \Lambda Q^{*}, \quad \Pi_{\pi} Q_{k}=\left[\frac{A}{B}\right], \mathcal{T}(A)=\mathbb{I}_{k}\right\}
$$

$\mathcal{M}((d-k) \times k)$ é o conjunto das matrizes $(d-k) \times k$. As cartas são definidas como

$$
\begin{aligned}
\Phi_{\pi}: U_{\pi} \subset G_{k}\left(\mathbb{R}^{d}\right) & \rightarrow \mathcal{M}((d-k) \times k) \\
E & \mapsto B A^{-1}
\end{aligned}
$$

A matriz $Q$, na decomposição espectral de $P_{E}$, não é única.

Proposição 4.3.1 A função $\Phi_{\pi}$ é bem definida.

Demonstração: Sejam $E \in U_{\pi}$ e $P_{E}$ a projeção ortogonal sobre $E$. Considere duas decomposições de $P_{E}$, ou seja, $P_{E}=Q \Lambda Q^{*}=\tilde{Q} \Lambda \tilde{Q}^{*} \operatorname{com} \Pi_{\pi} Q_{k}=$ $\left[\begin{array}{c}A \\ B\end{array}\right], \quad \Pi_{\pi} \tilde{Q}_{k}=\left[\begin{array}{c}\tilde{A} \\ \tilde{B}\end{array}\right]$ e $\mathcal{T}(A)=\mathcal{T}(\tilde{A})=\mathbb{I}_{k}$. Se na igualdade $Q \Lambda Q^{*}=$ $\tilde{Q} \Lambda \tilde{Q}^{*}$ multiplicamos $\tilde{Q}^{*}$ pela esquerda e $Q$ pela direita, temos

$$
W \Lambda=\Lambda W, \quad W=\tilde{Q}^{*} Q \in \mathcal{O}(d) .
$$


Se $W=\left[\begin{array}{l|l}W_{11} & W_{12} \\ \hline W_{21} & W_{22}\end{array}\right]_{d \times d}, \operatorname{com} W_{11} \in \mathcal{M}(k)$, então

$$
\begin{aligned}
& \begin{aligned}
& W \Lambda=\Lambda W \\
& {\left[\begin{array}{c|c}
W_{11} & W_{12} \\
\hline W_{21} & W_{22}
\end{array}\right]\left[\begin{array}{c|c}
I_{k \times k} & 0 \\
\hline 0 & 0
\end{array}\right]=\left[\begin{array}{c|c}
I_{k \times k} & 0 \\
\hline 0 & 0
\end{array}\right]\left[\begin{array}{c|c}
W_{11} & W_{12} \\
\hline W_{21} & W_{22}
\end{array}\right] }
\end{aligned} \\
& {\left[\begin{array}{c|c}
W_{11} & 0 \\
\hline W_{21} & 0
\end{array}\right]=\left[\begin{array}{c|c}
W_{11} & W_{12} \\
\hline 0 & 0
\end{array}\right] \text {. }}
\end{aligned}
$$

Logo $W_{12}=W_{21}=0$. Assim

$$
W=\left[\begin{array}{c|c}
W_{11} & 0 \\
\hline 0 & W_{22}
\end{array}\right]
$$

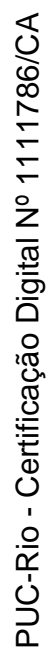

Multiplicando $\tilde{Q}$ pela esquerda na igualdade $W=\tilde{Q}^{*} Q$, temos

$$
Q=\tilde{Q} W
$$

Se $\tilde{Q}=\left[\begin{array}{c|c}\tilde{Q}_{11} & \tilde{Q}_{12} \\ \hline \tilde{Q}_{21} & \tilde{Q}_{22}\end{array}\right]$, então $\tilde{Q} W=\left[\begin{array}{c|c}\tilde{Q}_{11} W_{11} & \tilde{Q}_{12} W_{22} \\ \hline \tilde{Q}_{21} W_{11} & \tilde{Q}_{22} W_{22}\end{array}\right]$. Pondo

$Q=\left[\begin{array}{l|l}Q_{11} & Q_{12} \\ \hline Q_{21} & Q_{22}\end{array}\right]$, com $Q_{11} \in \mathcal{M}(k)$, a equação (4-6) implica

$$
\left[\begin{array}{l|l}
Q_{11} & Q_{12} \\
\hline Q_{21} & Q_{22}
\end{array}\right]=\left[\begin{array}{l|l}
\tilde{Q}_{11} W_{11} & \tilde{Q}_{12} W_{22} \\
\hline \tilde{Q}_{21} W_{11} & \tilde{Q}_{22} W_{22}
\end{array}\right]
$$

Portanto

$$
Q_{k}=\tilde{Q}_{k} W_{11}
$$

Multiplicando $\Pi_{\pi}$ pela direita, temos

$$
\begin{aligned}
\Pi_{\pi} Q_{k} & =\Pi_{\pi} \tilde{Q}_{k} W_{11} \\
{\left[\frac{A}{B}\right] } & =\left[\frac{\tilde{A} W_{11}}{\tilde{B} W_{11}}\right] .
\end{aligned}
$$

Isto é, $A=\tilde{A} W_{11}$ e $B=\tilde{B} W_{11}$. Como $W$ é ortogonal, da igualdade (4-5), 
concluímos que $W_{11}$ também é ortogonal e consequentemente inversível. Assim,

$$
B A^{-1}=\tilde{B} W_{11}\left(\tilde{A} W_{11}\right)^{-1}=\tilde{B} W_{11} W_{11}^{-1} \tilde{A}^{-1}=\tilde{B} \tilde{A}^{-1} .
$$

Portanto, $\Phi_{\pi}$ é bem definida. O seguinte diagrama revisa o procedimento feito

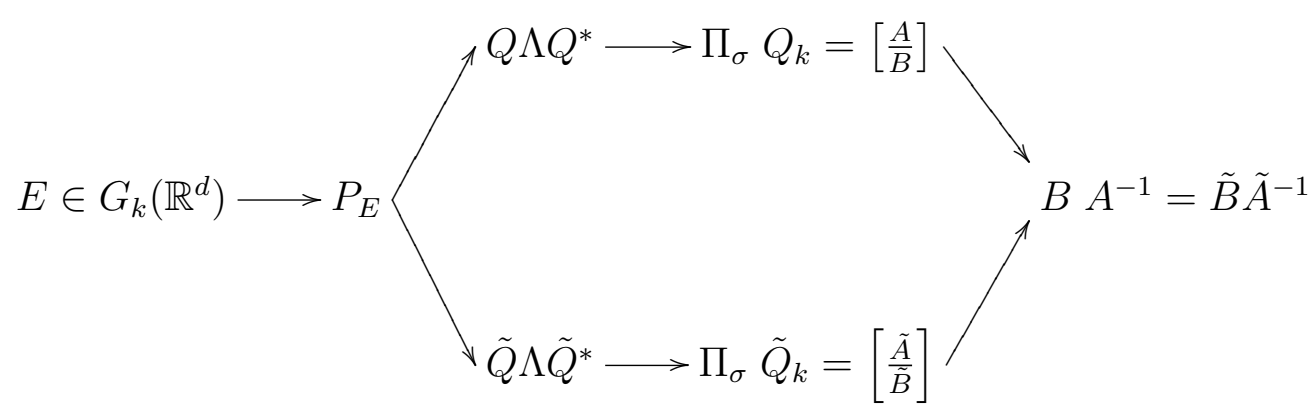

Agora mostraremos que $\Phi_{\pi}$ é bijetiva. Começamos com a injetividade. Sejam $P_{E}$ e $P_{F}$, respectivamente, duas projeções ortogonais sobre $E$ e $F \in U_{\pi}$. Suponha que $\Phi_{\pi}(E)=\Phi_{\pi}(F)$

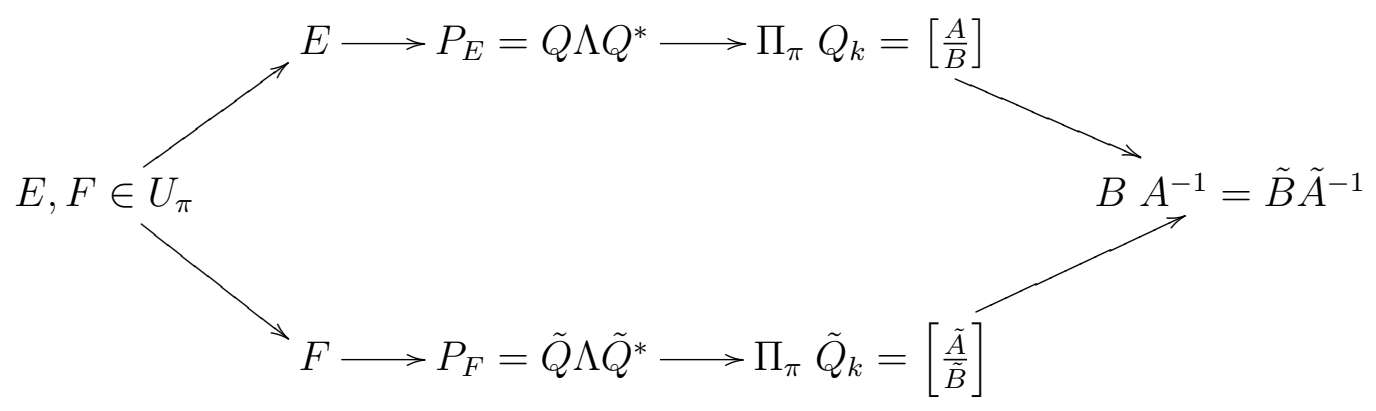

Tome $S=\tilde{A}^{-1} A$, então da igualdade $\tilde{B} \tilde{A}^{-1}=B A^{-1}$, temos $\tilde{B} S=$ $\tilde{B}\left(\tilde{A}^{-1} A\right)=\left(\tilde{B} \tilde{A}^{-1}\right) A=\left(B A^{-1}\right) A=B$. Além disso, $\tilde{A} S=A$. Logo

$$
\Pi_{\pi} Q_{k}=\left[\frac{A}{B}\right]=\left[\frac{\tilde{A} S}{\tilde{B} S}\right]=\left[\frac{\tilde{A}}{\tilde{B}}\right] S=\Pi_{\pi} \tilde{Q}_{k} S .
$$

Portanto, $Q_{k}=\tilde{Q}_{k} S$ - equivalentemente $Q_{k} S^{-1}=\tilde{Q}_{k}-$. Ou seja, cada coluna de $\tilde{Q}_{k}$ é combinação linear das colunas de $Q_{k}$. Lembramos que as colunas de $Q_{k}$ e $\tilde{Q}_{k}$ são, respectivamente, bases para $E$ e $F$. Logo, $E=F$. Assim, $\Phi_{\pi}$ é injetiva.

Agora mostraremos que $\Phi_{\pi}$ é sobrejetora. Seja $Z \in \mathcal{M}((d-k) \times k)$. 
Tomamos $I_{k}$, a matriz identidade $k \times k$, e fazemos $M=\left[\frac{I_{k}}{Z}\right]_{d \times k}$. Lembramos ao leitor que o processo de ortogonalização de Gram-Schmidt, aplicado às colunas de $M$, nos fornece uma matriz triangular superior $R_{k \times k}$, cujas entradas na diagonal são todas diferentes de 0 (logo inversível), e uma matriz ortogonal $\bar{Q}_{k}=\left[\frac{\bar{A}}{\bar{B}}\right]_{d \times k}$, tal que

$$
\left[\begin{array}{l}
I_{k} \\
Z
\end{array}\right]=\left[\frac{\bar{A}}{\bar{B}}\right][R]=\left[\begin{array}{c}
\bar{A} R \\
\bar{B} R
\end{array}\right]
$$

Ora, da igualdade $\bar{A} R=I_{k}$ segue que $\bar{A}=R^{-1}$. Dado que $R^{-1}$ também é triangular superior, com todas as entradas da diagonal não nulas, concluímos que $\mathcal{T}(\bar{A})=\mathbb{I}_{k}$.

Se $Q_{k}=\Pi_{\pi}^{-1} \bar{Q}_{k}$, então $Q_{k}$ é ortogonal, pois é produto de duas matrizes ortogonais. Tomamos, então, $E$ como sendo o subespaço vetorial gerado pelas colunas de $Q_{k}$. Desta maneira, temos $P_{E}=Q \Lambda Q^{*} \operatorname{com} Q=Q_{k} Q_{k}^{*} \in \mathcal{O}(d)$. Afirmamos que $\Phi_{J}(E)=Z$

$$
E \mapsto P_{E}=Q \Lambda Q^{*} \mapsto \Pi_{\pi} Q_{k}=\left[\begin{array}{c}
\bar{A} \\
\bar{B}
\end{array}\right] \mapsto \bar{B} \bar{A}^{-1}
$$

Usando (4-8), decretamos a sobrejetividade de $\Phi_{\pi}$

$$
\bar{B} \bar{A}^{-1}=\bar{B} R\left(R^{-1} \bar{A}^{-1}\right)=\bar{B} R(\bar{A} R)^{-1}=\bar{B} R I_{k}=\bar{B} R=Z .
$$

Finalmente, mostramos a diferenciabilidade das mudanças de coordenadas.

Proposição 4.3.2 Sejam $\Phi_{\pi}: U_{\pi} \rightarrow \mathcal{M}((d-k) \times k)$ e $\Phi_{\mu}: U_{\mu} \rightarrow$ $\mathcal{M}((d-k) \times k)$ duas cartas locais. Então a mudança de coordenadas

$$
\begin{aligned}
\Phi_{\mu} \circ \Phi_{\pi}^{-1}: \Phi_{\pi}\left(U_{\pi} \cap U_{\mu}\right) & \rightarrow \Phi_{\mu}\left(U_{\pi} \cap U_{\mu}\right) \\
Z & \mapsto \Phi_{\mu}\left(\Phi_{\pi}^{-1}(Z)\right)
\end{aligned}
$$

é suave. 
Demonstração: Denotemos $\Phi_{\pi \mu}=\Phi_{\mu} \circ \Phi_{\pi}^{-1}$. Sejam $Z \in \Phi_{\pi}\left(U_{\pi} \cap U_{\mu}\right)$ e $E=\Phi_{\pi}^{-1}(Z) \in U_{\pi} \cap U_{\mu}$. Seguindo exatamente o mesmo argumento feito para a matriz $Z$ em (4-8), tomamos $\bar{Q}_{k}=\left[\begin{array}{c}\bar{A} \\ \bar{B}\end{array}\right]$ e $Q_{k}=\Pi_{\pi}^{-1} \bar{Q}_{k}$. Dado que $E \in U_{\pi}$, temos $P_{E}=Q \Lambda Q^{*}$ tal que $\Pi_{\pi} Q_{k}=\left[\begin{array}{c}A \\ B\end{array}\right], \mathcal{T}(A)=\mathbb{I}_{k}$. Por outro lado, se $E \in U_{\mu}$, temos $P_{E}=\tilde{Q} \Lambda \tilde{Q}^{*}$ tal que $\Pi_{\mu} \tilde{Q}_{k}=\left[\frac{\tilde{A}}{\tilde{B}}\right], \mathcal{T}(\tilde{A})=\mathbb{I}_{k}$ e $\Phi_{\mu}(E)=\tilde{B} \tilde{A}^{-1}$. Lembramos também que as colunas de $Q_{k}$, assim como as de $\tilde{Q}_{k}$, formam uma base de $E$. Logo, existe uma matriz $B$ de dimensão $k \times k$ tal que, $\tilde{Q}_{k}=Q_{k} B$. Desta maneira, $\Phi_{\pi \mu}(\mathrm{Z})$ é dado por uma sequência de aplicações diferenciáveis

$$
Z \mapsto\left[\begin{array}{c}
I_{k} \\
Z
\end{array}\right]=\bar{Q} R \mapsto Q_{k}=\Pi_{\pi}^{-1} \bar{Q}_{k} \mapsto \tilde{Q}_{k}=Q_{k} B \mapsto \Pi_{\mu} \tilde{Q}_{k}=\left[\begin{array}{c}
\tilde{A} \\
\tilde{B}
\end{array}\right] \mapsto \tilde{B}^{-1}
$$




\section{5}

\section{O Teorema de Johnson-Lindenstrauss}

Enunciamos o teorema para familiarizar o leitor

Teorema 5.1 Fixe $\epsilon \in(0,1)$ e $d, n \in \mathbb{N}$. Tome $k \in \mathbb{N}$ tal que

$$
d>k \geq 4\left(\frac{\epsilon^{2}}{2}-\frac{\epsilon^{3}}{3}\right)^{-1} \ln n
$$

Então, para qualquer conjunto $V$ com $n$ pontos em $\mathbb{R}^{d}$, existe um subespaço $E \subset \mathbb{R}^{d}$ de dimensão $k$ para o qual temos que $\forall u, v \in V$

$$
(1-\epsilon)\|u-v\|^{2} \leq\left\|\sqrt{d / k}\left(\pi_{E}(u)-\pi_{E}(v)\right)\right\|^{2} \leq(1+\epsilon)\|u-v\|^{2},
$$

onde $\pi_{E}: \mathbb{R}^{d} \rightarrow E$ é a projeção ortogonal sobre $E$.

Nas primeiras duas secções vemos como o mecanismo push-forward nos permite induzir medidas uniformes em $S^{d-1}$ e $G_{k}\left(\mathbb{R}^{d}\right)$. ${ }^{1} \mathrm{Na}$ seção 3 , as distribuições gaussianas surgem a fim de obter um algoritmo capaz de escolher um ponto da esfera de modo uniforme. Além disso, a concentração de certas variáveis aleatórias segue estimando gaussianas. Surgem duas perspectivas: a primeira é projetar pontos aleatórios da esfera $S^{d-1} \mathrm{em} \mathbb{R}^{k}$ e a segunda, projetar um ponto fixo de $S^{d-1}$ em subespaços aleatórios de $G_{k}\left(\mathbb{R}^{d}\right)$. Estes dois pontos de vista são equivalentes. Finalmente na seção 4 demonstramos o teorema de Johnson-Lindenstrauss. Começamos com o coração deste procedimento: uma medida invariante em $\mathcal{O}(d)$.

\section{1}

\section{Medida de Haar em $\mathcal{O}(d)$}

Embora a medida de Haar exista para qualquer grupo localmente compacto, restringiremos a nossa atenção a grupos compactos. A existência e unicidade da medida de Haar não serão provadas.

${ }^{1}$ No capítulo 3 , a medida uniforme em $S^{d-1}$ é obtida normalizando a medida de Lebesgue em $S^{d-1}$ 
Sejam $G$ um grupo topológico compacto e $\mu$ uma medida definida nos borelianos de $G$. A medida $\mu$ é invariante se para todo boreliano $A \subset G$ e para todo $g \in G$, temos $\mu(A)=\mu\{g a: a \in A\}=\mu\left\{a g^{-1}: a \in A\right\}$.

A demonstração do seguinte teorema pode ser vista em (7, p. 78).

Teorema 5.2 (Haar) Se G é um grupo topológico compacto, então existe uma única medida invariante $\mu$ definida nos borelianos de $G$ com $\mu(G)=1$. Esta medida é a medida Haar do grupo $G$.

Corolário 5.3 Seja $\mathcal{O}(d)$ o grupo ortogonal. Existe uma única medida de Haar $\mu$ em $\mathcal{O}(d)$. Ou seja, para todo boreliano $A \subset G$ e para todo $g \in \mathcal{O}(d)$, temos

$$
\mu(A)=\mu\{g a: a \in A\}=\mu\left\{a g^{-1}: a \in A\right\} .
$$

Demonstração: O grupo $\mathcal{O}(d)$ é um grupo topológico compacto, assim, o resultado segue aplicando o Teorema 5.2.

A seguir, construiremos medidas invariantes de uma maneira geral, ou seja, em qualquer variedade. Considere $\mathcal{O}(d)$ e $\mu$ sua medida de Haar. Sejam $M$ uma variedade e $\phi: \mathcal{O}(d) \times M \rightarrow M$ uma ação transitiva. Fixando um ponto $x \in M$ arbitrário, definimos a função $\phi_{x}: \mathcal{O}(d) \rightarrow M, g \mapsto \phi_{x}(g, x)=g x$. Induzimos a medida $\vartheta$, como sendo o push-forward da medida de Haar $\mu$. Isto é, para todo boreliano $A \subset M$

$$
\vartheta(A):=\mu\left(\phi_{x}^{-1}(A)\right)=\mu\{g \in \mathcal{O}(d): g x \in A\} .
$$

Teorema 5.4 A medida $\vartheta$ definida em $M$ é a única medida de probabilidade invariante pela ação de $\mathcal{O}(d) .^{2}$

Demonstração: Provaremos primeiro a invariância de $\vartheta$. Sejam $g \in \mathcal{O}(d)$ e $A \subset M$ um boreliano. Da invariância de $\mu$, temos $\mu\left(\phi_{x}^{-1}(A)\right)=\mu\left(g \phi_{x}^{-1}(A)\right)$. Se $g\left(\phi_{x}^{-1}(A)\right)=\phi_{x}^{-1}(g A)$, então $\mu\left(\phi_{x}^{-1}(A)\right)=\mu\left(g\left(\phi_{x}^{-1}(A)\right)\right)=\mu\left(\phi_{x}^{-1}(g A)\right)$. Ou seja, $\vartheta(A)=\vartheta(g A)$. Assim, a invariância de $\vartheta$ se resume a provar a igualdade $g\left(\phi_{x}^{-1}(A)\right)=\phi_{x}^{-1}(g A), \forall g \in \mathcal{O}(d)$ e $\forall A \subset M$ boreliano. Veja o diagrama abaixo.
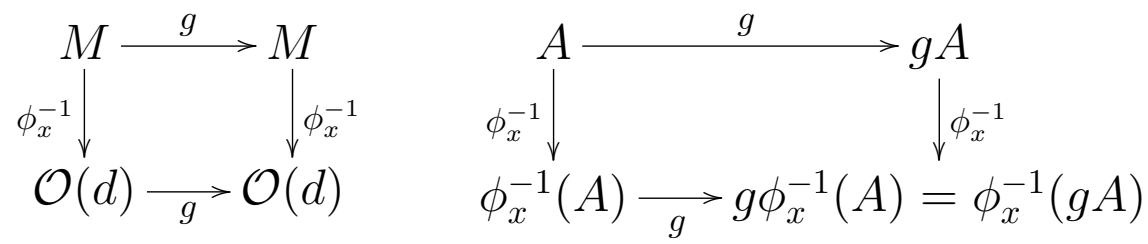

\footnotetext{
${ }^{2}$ Lembramos ao leitor que a medida $\vartheta$ é invariante pela ação do grupo $\mathcal{O}(d)$ se para todo
} $g \in \mathcal{O}(d)$ e para todo conjunto boreliano $A \subset M$, temos $\vartheta(A)=\vartheta(g A)$. 
Lembre que se $B \subset M$ é um conjunto qualquer, então $h \in \phi_{x}^{-1}(B)$ se, e somente se, $\phi(h, x)=h x \in B$. Provaremos primeiro que $g \phi_{x}^{-1}(A) \subset \phi_{x}^{-1}(g A)$. Se $\tilde{h}=g h \in g \phi_{x}^{-1}(A)$, com $h \in \phi_{x}^{-1}(A)$, i.e., $\phi(h, x)=h x \in A$, então $\phi(g h, x)=g h x \in g A$. Logo $\tilde{h} \in \phi_{x}^{-1}(g A)$. Reciprocamente, se $\tilde{h} \in \phi_{x}^{-1}(g A)$, então $\phi(\tilde{h}, x) \in g A$, i.e., existe $a \in A$ tal que $\phi(\tilde{h}, x)=\tilde{h} x=g a$. Provar que $\tilde{h} \in g \phi_{x}^{-1}(A)$, equivale a provar que $g^{-1} \tilde{h} \in \phi_{x}^{-1}(A)$, i.e., $\phi\left(g^{-1} \tilde{h}, x\right) \in A$. De fato, $g^{-1} \tilde{h} x=g^{-1} g a=a \in A$. Logo, $\phi_{x}^{-1}(g A) \subset g \phi_{x}^{-1}(A)$. Provando a invariância de $\vartheta$.

É claro que $\mathcal{O}(d)=\phi_{x}^{-1}(M)$. Logo $\vartheta(M)=1$. Assim, $\vartheta$ é uma medida de probabilidade.

Resta provar a unicidade. Suponha $\tilde{\vartheta}$ outra medida de probabilidade em $M$ invariante pela ação do grupo $\mathcal{O}(d)$. Defina a medida $\tilde{\mu}$ em $\mathcal{O}(d)$ da seguinte maneira: se $S$ é um boreliano de $\mathcal{O}(d)$, então

$$
\tilde{\mu}(S):=\tilde{\vartheta}\left(\phi_{x}(S)\right)=\tilde{\vartheta}\left(\left\{\phi_{x}(h): h \in S\right\}\right)
$$

Lembre que a ação é transitiva, i.e., $\forall y \in M, \exists g \in \mathcal{O}(d)$ tal que $\phi_{x}(g)=y$. Ou seja, $\phi_{x}$ é sobrejetiva. Assim, $\phi_{x}\left(\phi_{x}^{-1}(A)\right)=A, \forall A \subset M$ boreliano e $\tilde{\mu}\left(\phi_{x}^{-1}(A)\right)=\tilde{\vartheta}(A)$. Mais ainda, da sobrejetividade da função $\phi_{x}$, temos $\phi_{x}(\mathcal{O}(d))=M$. Isto é $\tilde{\mu}(\mathcal{O}(d))=1$. Suponha $\tilde{\mu}$ invariante. Pela unicidade de $\mu$ em $\mathcal{O}(d)$, temos $\tilde{\mu}=\mu$. Portanto, $\tilde{\vartheta}(A)=\tilde{\mu}\left(\phi_{x}^{-1}(A)\right)=\mu\left(\phi_{x}^{-1}(A)\right)=\vartheta(A)$. Assim, a unicidade de $\tilde{\vartheta}$ se resume à invariância de $\tilde{\mu}$.

Novamente, da invariância de $\tilde{\vartheta}$, temos $\tilde{\vartheta}\left(\phi_{x}(S)\right)=\tilde{\vartheta}\left(g \phi_{x}(S)\right)$ e supondo $g \phi_{x}(S)=\phi_{x}(g S), \forall g \in \mathcal{O}(d)$ e $\forall S \subset \mathcal{O}(d)$ subconjunto boreliano, temos $\tilde{\vartheta}\left(\phi_{x}(S)\right)=\tilde{\vartheta}\left(g \phi_{x}(S)\right)=\tilde{\vartheta}\left(\phi_{x}(g S)\right.$. Concluindo $\tilde{\mu}(S)=\tilde{\mu}(g S)$. Logo, provar a invariância de $\tilde{\mu}$ equivale a provar $g \phi_{x}(S)=\phi_{x}(g S)$
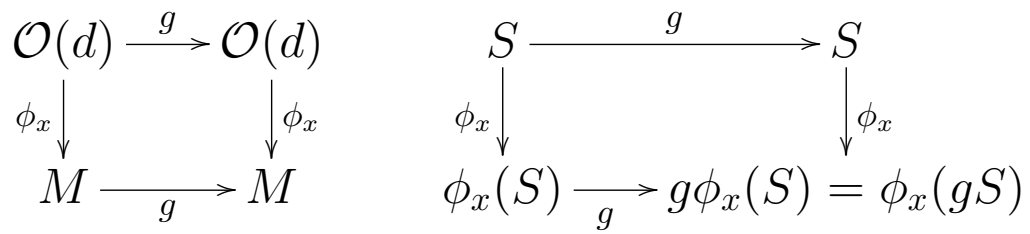

Mas este fato é imediato, uma vez que $\forall h \in S \subset \mathcal{O}(d)$, temos

$$
g \phi_{x}(h)=g \phi(h, x)=\phi(g, \phi(h, x))=\phi(g h, x)=\phi_{x}(g h) .
$$

Portanto, a invariância de $\tilde{\mu}$ está provada. Resumindo, se existe outra medida invariante $\tilde{\vartheta}$ em $M$ com $\tilde{\vartheta}(M)=1$, então $\tilde{\vartheta}=\vartheta$.

O corolário a seguir afirma que a medida $\vartheta$ em $M$ pode ser definida de outra maneira. 
Corolário 5.5 A medida $\vartheta$ é tal que

$$
\vartheta(A)=\mu\left\{g \in \mathcal{O}(d): g^{-1} x \in A\right\}
$$

Demonstração: Defina $\bar{\vartheta}(A):=\mu\left\{g \in \mathcal{O}(d): g^{-1} x \in A\right\}$. Provaremos que $\bar{\vartheta}$ é invariante pela ação do grupo ortogonal. Tome um boreliano $A \subset M \mathrm{e}$ $h \in \mathcal{O}(d)$. Da invariância da medida de Haar $\mu$ exibida em (5-1), temos

$$
\bar{\vartheta}(A)=\mu\left\{g \in \mathcal{O}(d): g^{-1} x \in A\right\}=\mu\left\{g h^{-1} \in \mathcal{O}(d): g^{-1} x \in A\right\}
$$

Afirmamos que $\left\{g h^{-1} \in \mathcal{O}(d): g^{-1} x \in A\right\}=\left\{g^{\prime} \in \mathcal{O}(d):\left(g^{\prime}\right)^{-1} x \in h A\right\}$. De fato, se $g^{\prime}=g h^{-1} \operatorname{com} g^{-1} x \in A$, então $\left(g^{\prime}\right)^{-1} x=\left(g h^{-1}\right)^{-1} x=h g^{-1} x \in h A$. Assim, $\left\{g h^{-1} \in \mathcal{O}(d): g^{-1} x \in A\right\} \subset\left\{g^{\prime} \in \mathcal{O}(d):\left(g^{\prime}\right)^{-1} x \in h A\right\}$.

Reciprocamente, tome $g^{\prime} \in \mathcal{O}(d)$ tal que $\left(g^{\prime}\right)^{-1} x \in h A$, então $g^{\prime}=$ $\left(g^{\prime} h\right) h^{-1}$ e $\left(g^{\prime} h\right)^{-1} x=h^{-1} g^{\prime} x \in A$. Logo, $\left\{g h^{-1} \in \mathcal{O}(d): g^{-1} x \in A\right\} \supset$ $\left\{g^{\prime} \in \mathcal{O}(d):\left(g^{\prime}\right)^{-1} x \in h A\right\}$. Portanto,

$$
\bar{\vartheta}(A)=\mu\left\{g^{\prime} \in \mathcal{O}(d):\left(g^{\prime}\right)^{-1} x \in h A\right\}=\bar{\vartheta}(h A) .
$$

Isto é, $\bar{\vartheta}$ é invariante pela ação do grupo $\mathcal{O}(d)$. É claro que $\bar{\vartheta}(M)=1$. Assim, da unicidade da medida $\vartheta$, concluímos que $\vartheta(A)=\bar{\vartheta}(A)$.

\section{2}

\section{Medidas invariantes em $S^{d-1}$ e em $G_{k}\left(\mathbb{R}^{d}\right)$}

Sejam $\phi: \mathcal{O}(d) \times S^{d-1} \rightarrow S^{d-1},(g, x) \mapsto g x$ a ação dada no capítulo 2 e $\mu$ a medida de Haar em $\mathcal{O}(d)$. Fixemos um ponto qualquer $s$ em $S^{d-1}$. Considere a aplicação $\phi_{s}: \mathcal{O}(d) \rightarrow S^{d-1}, g \mapsto g s$. Definimos a medida $\sigma$ em $S^{d-1}$ pondo para cada boreliano $A \subset S^{d-1}$

$$
\sigma(A):=\mu\left(\phi_{s}^{-1}(A)\right)=\mu\{g \in \mathcal{O}(d): g s \in A\}
$$

A ação $\phi$ é transitiva. De fato, tome $a, b$ em $S^{d-1}$. A partir do vetor $a$, completamos uma base $\alpha=\left\{a_{1}=a, a_{2}, a_{3}, \cdots, a_{d}\right\}$ de $\mathbb{R}^{d}$ e pelo processo de Gram-Schmidt, podemos garantir a ortonormalidade de $\alpha$. De maneira análoga, partindo de $b \in S^{d-1}$ obtemos uma base ortonormal $\beta=\left\{b_{1}=b, b_{2}, b_{3}, \cdots, b_{d}\right\}$ de $\mathbb{R}^{d}$. Definimos o operador $g: \mathbb{R}^{d} \rightarrow \mathbb{R}^{d}$ tal que $a_{i} \mapsto b_{i}$ para todo $i=1, \cdots, d$. Logo $g \in \mathcal{O}(d)$ e $g a=b$. 
Pelo Teorema 5.4 concluímos que $\sigma$ é a única medida invariante pela ação do grupo $\mathcal{O}(d)$. Além disso, pelo Corolário 5.5

$$
\sigma(A)=\mu\left\{g \in \mathcal{O}(d): g^{-1} s \in A\right\}
$$

Analogamente, induzimos uma medida em $G_{k}\left(\mathbb{R}^{d}\right)$. Sejam $\mathcal{O}(d)$ o grupo ortogonal e $G_{k}\left(\mathbb{R}^{d}\right)$ a Grassmannianas. Se $g \in \mathcal{O}(d)$ e $E \in G_{k}\left(\mathbb{R}^{d}\right)$, então $g E$ é a imagem de $E$ pela matriz $g$. Tome a ação $\phi: \mathcal{O}(d) \times G_{k}\left(\mathbb{R}^{d}\right) \rightarrow G_{k}\left(\mathbb{R}^{d}\right)$, $(g, E) \mapsto g E$ e aplicação $\phi_{\mathbb{R}^{k}}: \mathcal{O}(d) \rightarrow G_{k}\left(\mathbb{R}^{d}\right), g \mapsto g \mathbb{R}^{k}$. Para todo boreliano $A \subset G_{k}\left(\mathbb{R}^{d}\right)$ definimos a medida $\xi$

$$
\xi(A)=: \mu\left\{g \in \mathcal{O}(d): g \mathbb{R}^{k} \in A\right\} .
$$

A prova da transitividadade de $\xi$ é análoga ao caso da medida $\sigma$ em $S^{d-1}$. Logo, a medida $\xi$ é a única medida nas Grasmannianas invariante pela ação do grupo $\mathcal{O}(d)$.

\section{3}

\section{Gaussianas e o lema geométrico}

Dizemos que uma variável aleatória $X$ tem distribuição Gaussiana univariada, $X \backsim N(0,1)$, se $X$ tem a função densidade

$$
f(x)=\frac{1}{\sqrt{2 \pi}} \exp \left(-\frac{x^{2}}{2}\right)
$$

Analogamente, um vetor aleatório $X=\left(X_{1}, X_{2} \cdots, X_{d}\right)$ tem distribuição Gaussiana multivariada, $X \backsim N(0, I)$, se cada variável aleatória $X_{i}$ é tal que $X_{i} \backsim N(0,1)$ e todas são mutuamente independentes. Neste caso, a função densidade de $X$ é

$$
f(x)=\frac{1}{(2 \pi)^{d / 2}} \exp \left(\frac{-\|x\|^{2}}{2}\right) .
$$

Lembramos que a distribuição de $X=\left(X_{1}, X_{2}, \cdots, X_{d}\right)$ é uma medida de probabilidade definida nos borelianos de $\mathbb{R}^{d}$. Usando a sua função densidade, a distribuição de $X$ é dada por

$$
\operatorname{Pr}[X \in B]=\frac{1}{(2 \pi)^{d / 2}} \int_{B} \exp \left(\frac{-\|x\|^{2}}{2}\right) d x, \quad \forall B \in \mathcal{B}\left(\mathbb{R}^{d}\right) .
$$

Proposição 5.3.1 Se $X=\left(X_{1}, X_{2} \cdots, X_{d}\right)$ tem distribuição Gaussiana multivariada, então sua distribuição é invariante pela ação do grupo $\mathcal{O}(d)$. Ou 
seja, $\forall B \in \mathcal{B}\left(\mathbb{R}^{d}\right)$ e $\forall g \in \mathcal{O}(d)$, temos

$$
\operatorname{Pr}[X \in B]=\operatorname{Pr}[X \in g B] .
$$

Demonstração: Faça $\alpha=\frac{1}{(2 \pi)^{d / 2}}$. Assim,

$$
\begin{aligned}
\operatorname{Pr}[X \in g B] & =\alpha \int_{g B} \exp \left(\frac{-\|x\|^{2}}{2}\right) d x=\alpha \int_{B} \exp \left(\frac{-\|g y\|^{2}}{2}\right)\left|\operatorname{det}\left(g^{\prime}(y)\right)\right| d y \\
& =\alpha \int_{B} \exp \left(\frac{-\|g y\|^{2}}{2}\right)|\operatorname{det}(g)| d y=\alpha \int_{B} \exp \left(\frac{-\|g y\|^{2}}{2}\right) d y \\
& =\alpha \int_{B} \exp \left(\frac{-\|y\|^{2}}{2}\right) d y=\operatorname{Pr}[X \in B]
\end{aligned}
$$

Dizemos que um vetor aleatório $Y$ tem distribuição uniforme em $S^{d-1}$ se sua distribuição é dada por

$$
\operatorname{Pr}[Y \in A]=\frac{\eta(A)}{\eta\left(S^{d-1}\right)}=\sigma(A), \quad \forall A \in \mathcal{B}\left(S^{d-1}\right)
$$

Considere a função mensurável $f: \mathbb{R}^{d} \backslash\{0\} \rightarrow S^{d-1}, x \mapsto \frac{x}{\|x\|}$ e o vetor $X=\left(X_{1}, X_{2} \cdots, X_{d}\right)$ com distribuição multivariada. Definimos o vetor aleatório $Y=\frac{1}{\|X\|}\left(X_{1}, X_{2}, \cdots, X_{d}\right)$. A distribuição de $Y$ é dada por

$$
\operatorname{Pr}[Y \in A]=\operatorname{Pr}\left[X \in f^{-1}(A)\right], \quad \forall A \in \mathcal{B}\left(S^{d-1}\right)
$$

O seguinte resultado afirma que $Y$ significa escolher um ponto aleatório da esfera de modo uniforme.

Proposição 5.3.2 $Y=\frac{1}{\|X\|}\left(X_{1}, X_{2}, \cdots, X_{d}\right)$ tem distribuição uniforme em $S^{d-1}$.

Demonstração: A igualdade (5-6) e a Proposição 5.3.1 implicam, respectivamente, as duas primeiras igualdades a seguir

$$
\operatorname{Pr}[Y \in A]=\operatorname{Pr}\left[X \in f^{-1}(A)\right]=\operatorname{Pr}\left[X \in g f^{-1}(A)\right]=\operatorname{Pr}\left[X \in f^{-1}(g A)\right]
$$

E a última igualdade é consequência de $g f^{-1}(A)=f^{-1}(g A)$. Logo, usando novamente (5-6), concluímos que $\operatorname{Pr}[Y \in A]=\operatorname{Pr}[Y \in g A]$.

Ao projetarmos o vetor $Y$ em suas $k$ primeiras coordenadas obtemos $Z=\frac{1}{\|X\|}\left(X_{1}, X_{2}, \cdots, X_{k}\right)$. Ou seja, $Z$ é a projeção em $\mathbb{R}^{k}$ de um ponto da esfera escolhido de maneira uniforme. Seja a variável aleatória $L: S^{d-1} \rightarrow \mathbb{R}$, 
$L=\|Z\|^{2}$. A distribuição de $L$ na reta é dada por

$$
\operatorname{Pr}[L \in B]=\sigma\left\{x \in S^{d-1}:\left\|\pi_{\mathbb{R}^{k}}(x)\right\|^{2} \in B\right\}, \quad \forall B \in \mathcal{B}(\mathbb{R})
$$

Note que pela independência das coordenadas de $Z$ e do fato $X_{i} \backsim N(0,1)$, temos $\mu_{i}=\mathbb{E}\left[\frac{X_{i}^{2}}{\left(X_{1}^{2}+\cdots,+X_{d}^{2}\right)}\right]=1 / d$. Logo, $\mathbb{E}[L]=\sum_{i=1}^{i=k} \mu_{i}=k / d$. Mostraremos que a variável aleatória $L$ se encontra fortemente concentrada em torno de sua média.

Lema 5.6 Seja $k<d$. Temos então

a. Se $0<\beta<1$, então

$$
\operatorname{Pr}\left[L \leq \beta \frac{k}{d}\right] \leq \beta^{\frac{k}{2}}\left(1+\frac{(1-\beta) k}{(d-k)}\right)^{\frac{(d-k)}{2}} \leq \exp \left(\frac{k}{2}(1-\beta+\ln \beta)\right)
$$

b. Se $1<\beta<d / k$, então

$$
\operatorname{Pr}\left[L \geq \beta \frac{k}{d}\right] \leq \beta^{\frac{k}{2}}\left(1+\frac{(1-\beta) k}{(d-k)}\right)^{\frac{(d-k)}{2}} \leq \exp \left(\frac{k}{2}(1-\beta+\ln \beta)\right) .
$$

Demonstração: Note que

$$
L=\|Z\|^{2}=\frac{X_{1}^{2}+X_{2}^{2}+\cdots+X_{k}^{2}}{X_{1}^{2}+X_{2}^{2}+\cdots+X_{d}^{2}}
$$

Assim,

$$
\operatorname{Pr}\left[L \leq \beta \frac{k}{d}\right]=\operatorname{Pr}\left[d\left(X_{1}^{2}+X_{2}^{2}+\cdots+X_{k}^{2}\right) \leq k \beta\left(X_{1}^{2}+X_{2}^{2}+\cdots+X_{d}^{2}\right)\right]
$$

Manipulando o lado direito desta igualdade, temos

$$
\operatorname{Pr}\left[L \leq \beta \frac{k}{d}\right]=\operatorname{Pr}\left[d \sum_{i=1}^{k} X_{i}^{2} \leq k \beta \sum_{i=1}^{d} X_{i}^{2}\right]=\operatorname{Pr}\left[k \beta \sum_{i=1}^{d} X_{i}^{2}-d \sum_{i=1}^{k} X_{i}^{2} \geq 0\right] .
$$

Para $t>0$, temos 


$$
\begin{aligned}
& \operatorname{Pr}\left[k \beta \sum_{i=1}^{d} X_{i}^{2}-d \sum_{i=1}^{k} X_{i}^{2} \geq 0\right]=\operatorname{Pr}\left[\exp \left\{t\left(k \beta \sum_{i=1}^{d} X_{i}^{2}-d \sum_{i=1}^{k} X_{i}^{2}\right)\right\} \geq 1\right] \\
& \leq \mathbb{E}\left[\exp \left\{t\left(k \beta \sum_{i=1}^{d} X_{i}^{2}-d \sum_{i=1}^{k} X_{i}^{2}\right)\right\}\right] \quad \text { Desigualdade de Markov } \\
& =\mathbb{E}\left[\exp \left\{t(k \beta-d) \sum_{i=1}^{k} X_{i}^{2}+t k \beta \sum_{i=k+1}^{d} X_{i}^{2}\right\}\right] \\
& =\mathbb{E}\left[\exp \left\{t(k \beta-d) X^{2}\right\}\right]^{k} \mathbb{E}\left[\exp \left\{t k \beta X^{2}\right\}\right]^{d-k} . \quad(X \sim N(0,1))
\end{aligned}
$$

Logo

$$
\operatorname{Pr}\left[L \leq \beta \frac{k}{d}\right] \leq \mathbb{E}\left[\exp \left\{t(k \beta-d) X^{2}\right\}\right]^{k} \mathbb{E}\left[\exp \left\{t k \beta X^{2}\right\}\right]^{d-k}, \quad t>0
$$

Lembremos a Proposição B.0.1 do Apêndice B: se a variável aleatória $X$ tem distribuição normal univariada, então

$$
\mathbb{E}\left[\exp \left(s X^{2}\right)\right]=1 / \sqrt{1-2 s}, \quad-\infty<s<1 / 2
$$

Logo, se $t(k \beta-d)<1 / 2$ e $t k \beta<1 / 2$, então

$\mathbb{E}\left[\exp \left\{t(k \beta-d) X^{2}\right\}\right]^{k} \mathbb{E}\left[\exp \left\{t k \beta X^{2}\right\}\right]^{d-k}=(1-2 t k \beta)^{-(d-k) / 2}(1-2 t(k \beta-d))^{-k / 2}$

As restrições $t>0, t(k \beta-d)<1 / 2$ e $t k \beta<1 / 2$ implicam $0<t<1 / 2 k \beta$. Logo, se $g(t)=(1-2 t k \beta)^{-(d-k) / 2}(1-2 t(k \beta-d))^{-k / 2}$ então

$$
\operatorname{Pr}\left[L \leq \frac{\beta k}{d}\right] \leq g(t), \quad 0<t<1 / 2 k \beta
$$

Em particular, podemos escolher $t_{0} \in(0,1 / 2 k \beta)$ tal que $g\left(t_{0}\right) \leq g(t)$, $\forall t \in(0,1 / 2 k \beta)$. Assim, teríamos $\operatorname{Pr}\left[L \leq \frac{\beta k}{d}\right] \leq g\left(t_{0}\right)$. Nosso objetivo então é minimizar a função $g$. Note que

$$
g(t)=\frac{1}{\sqrt{f(t)}}, \quad f(t)=(1-2 t k \beta)^{(d-k)}(1-2 t(k \beta-d))^{k}
$$

Ainda, se $f\left(t_{0}\right) \geq f(t), \forall t \in(0,1 / 2 k \beta)$ então $g\left(t_{0}\right) \leq g(t), \forall t \in(0,1 / 2 k \beta)$. Logo, a fim de minimizar $g$, maximizamos $f$. Começamos derivando $f$ e para tal chame $c_{1}=(1-2 t k \beta)$ e $c_{2}=(1-2 t(k \beta-d))$. Assim, $f^{\prime}(t)=0$ fica

$$
\begin{aligned}
f^{\prime}(t) & =(d-k) c_{1}^{(d-k-1)} c_{2}^{k}(-2 k \beta)-k c_{1}^{(d-k)} c_{2}^{(k-1)} 2(d-k \beta) \\
& =2 k c_{1}^{(d-k-1)} c_{2}^{(k-1)}\left\{\left[-d \beta c_{2}-\beta k c_{1}\right]+\left[k \beta c_{2}+d c_{1}\right]\right\}=0
\end{aligned}
$$

Ora, observe que para $t \in(0,1 / 2 k \beta), 2 k c_{1}^{(d-k-1)} c_{2}^{(k-1)}>0$. Então 
$f^{\prime}(t)=0$ implica que

$$
\left[-d \beta c_{2}-\beta k c_{1}\right]+\left[k \beta c_{2}+d c_{1}\right]=0
$$

Substituindo $c_{1}$ e $c_{2}$ temos

$$
\left[-d \beta(1-2 t k \beta+2 t d)-\beta k+2 t k^{2} \beta^{2}\right]+[k \beta-k \beta 2 t(k \beta-d)+d-2 d t k \beta]=0 .
$$

Manipulando mais ainda, chegamos a $d(1-\beta)-2 d \beta(d-k \beta) t=0$. Assim, obtemos o único ponto crítico de $f$ em $(0,1 / 2 k \beta)$

$$
t_{0}=\frac{1-\beta}{2 \beta(d-k \beta)}
$$

Além disso,

$$
\begin{aligned}
f\left(t_{0}\right) & =\left(1-2\left(\frac{1-\beta}{2 \beta(d-k \beta)}\right) k \beta\right)^{(d-k)}\left(1-2\left(\frac{1-\beta}{2 \beta(d-k \beta)}\right)(k \beta-d)\right)^{k} \\
& =\left(1-\left(\frac{k-k \beta}{d-k \beta}\right)\right)^{(d-k)}\left(1+\left(\frac{1-\beta}{\beta}\right)\right)^{k} \\
& =\left(\frac{d-k}{d-k \beta}\right)^{(d-k)}\left(\frac{1}{\beta}\right)^{k} .
\end{aligned}
$$

Para provar que $t_{0}$ maximiza a função $f$ em $(0,1 / 2 k \beta)$ observamos que $\lim _{t \rightarrow 0^{+}} f(t)=1$ e $\lim _{t \rightarrow 1 / 2 k \beta^{-}} f(t)=0$. Estendemos $f$ em $[0,1 / 2 k \beta]$ fazendo $f(0)=1$ e $f(1 / 2 k \beta)=0$. Deste modo, obtemos uma função contínua definida em um compacto. Pelo Teorema de Weierstrass, a função extendida atinge seu máximo em um ponto de $[0,1 / 2 k \beta]$. Existe a possibilidade desse ponto ser o extremo esquerdo do intervalo $[0,1 / 2 k \beta]$. Descartamos esta possibilidade provando que $f(0)=1<f\left(t_{0}\right)$, onde $t_{0}$ é dado acima. Isto é, iremos provar que

$$
1<\left(\frac{d-k}{d-k \beta}\right)^{(d-k)}\left(\frac{1}{\beta}\right)^{k} .
$$

Definimos $P(\beta)=\frac{1}{(d-k)^{d-k}}(d-\beta k)^{d-k} \beta^{k}, \forall \beta \in(0, d / k)$. Assim, a desigualdade (5-9) equivale a $P(\beta)<P(1)$. Portanto, nossa tarefa é provar que $P$ atinge seu máximo estrito em $\beta=1$. Derivamos $P$

$$
P^{\prime}(\beta)=\frac{1}{(d-k)^{d-k}} k \beta^{k-1}(d-\beta k)^{d-k-1} d(1-\beta) .
$$

Observamos que para $0<\beta<1$ temos $P^{\prime}(\beta)>0$ e para $1<\beta<d / k$, $P^{\prime}(\beta)<0$. Isto implica que se $\beta \in(0,1)$, então $P$ é estritamente crescente e se $\beta \in(1, d / k)$, então $P$ é estritamente decrescente. Concluindo assim que $\beta=1$ 
é um ponto de máximo estrito.

Ora, tudo isto conclui que $f(t)<f\left(t_{0}\right), \forall t \in[0,1 / 2 k \beta]$. Em particular, $t_{0}$ maximiza $f$ em $(0,1 / 2 k \beta)$. Finalmente, usando $g\left(t_{0}\right)=\frac{1}{\sqrt{f\left(t_{0}\right)}}$, temos que

$$
\operatorname{Pr}\left[L \leq \beta \frac{k}{d}\right] \leq \beta^{k / 2}\left(\frac{d-k \beta}{d-k}\right)^{(d-k) / 2}
$$

A desigualdade $1+x \leq \exp (x), \forall x \in \mathbb{R}$ implica $\ln (1+x) \leq x, \forall x>-1$. Logo, dado que $\frac{(1-\beta) k}{(d-k)}>-1$, temos,

$$
\begin{aligned}
\ln \left(1+\frac{(1-\beta) k}{(d-k)}\right) & \leq \frac{(1-\beta) k}{(d-k)} \\
\frac{(d-k)}{2} \ln \left(1+\frac{(1-\beta) k}{(d-k)}\right) & \leq \frac{k}{2}(1-\beta) \\
\frac{k}{2} \ln \beta+\ln \left(1+\frac{(1-\beta) k}{(d-k)}\right)^{\frac{(d-k)}{2}} & \leq \frac{k}{2}+\frac{k}{2} \ln \beta-\frac{k}{2} \beta \\
\ln \beta^{\frac{k}{2}}+\ln \left(1+\frac{(1-\beta) k}{(d-k)}\right)^{\frac{(d-k)}{2}} & \leq \frac{k}{2}(1-\beta+\ln \beta) \\
\ln \left[\beta^{\left.\frac{k}{2}\left(1+\frac{(1-\beta) k}{(d-k)}\right)^{\frac{(d-k)}{2}}\right]}\right. & \leq \frac{k}{2}(1-\beta+\ln \beta) \\
\beta^{\frac{k}{2}}\left(1+\frac{(1-\beta) k}{(d-k)}\right)^{\frac{(d-k)}{2}} & \leq \exp \left(\frac{k}{2}(1-\beta+\ln \beta)\right) .
\end{aligned}
$$

Concluindo o primeiro item

$$
\operatorname{Pr}\left[L \leq \beta \frac{k}{d}\right] \leq \beta^{\frac{k}{2}}\left(1+\frac{(1-\beta) k}{(d-k)}\right)^{\frac{(d-k)}{2}} \leq \exp \left(\frac{k}{2}(1-\beta+\ln \beta)\right)
$$

Agora provaremos o item $b$, onde $\beta>1$.

$\operatorname{Pr}\left[L \geq \beta \frac{k}{d}\right]=\operatorname{Pr}\left[d\left(X_{1}^{2}+X_{2}^{2}+\cdots+X_{k}^{2}\right) \geq k \beta\left(X_{1}^{2}+X_{2}^{2}+\cdots+X_{d}^{2}\right)\right]$

Análogo ao primeiro item, manipulamos o lado direito desta equação. Assim,

$$
\operatorname{Pr}\left[L \geq \beta \frac{k}{d}\right] \leq \mathbb{E}\left[\exp \left\{t(d-k \beta) X^{2}\right\}\right]^{k} \mathbb{E}\left[\exp \left\{-t k \beta X^{2}\right\}\right]^{d-k}, \quad t>0
$$

Observe que $t>0, t(d-k \beta)<1 / 2 \mathrm{e}-t k \beta<1 / 2$ implica $0<t<1 / 2(d-k \beta)$. Logo, usando (5-8), temos

$\mathbb{E}\left[\exp \left\{t(d-k \beta) X^{2}\right\}\right]^{k} \mathbb{E}\left[\exp \left\{-t k \beta X^{2}\right\}\right]^{d-k}=(1+2 t k \beta)^{-(d-k) / 2}(1+2 t(k \beta-d))^{-k / 2}$ 
Portanto, $\forall t \in(0,1 / 2(d-k \beta))$

$$
\operatorname{Pr}\left[L \geq \beta \frac{k}{d}\right] \leq(1+2 t k \beta)^{-(d-k) / 2}(1+2 t(k \beta-d))^{-k / 2}=g(-t),
$$

onde $g(t)=(1-2 t k \beta)^{-(d-k) / 2}(1-2 t(k \beta-d))^{-k / 2}$. Novamente, nosso proposito é minimizar $g(-t)$ em $(0,1 / 2(d-k \beta))$. O procedimento é completamente análogo ao primeiro item: a fim de minimizar a função $g(-t)$ em $(0,1 / 2(d-k \beta))$, fazemos

$$
g(-t)=\frac{1}{\sqrt{f(t)}}, \quad f(t)=(1+2 t k \beta)^{(d-k)}(1+2 t(k \beta-d))^{k}
$$

e maximizamos $f$ em $(0,1 / 2(d-k \beta))$. Começamos fazendo $f^{\prime}(t)=0$

$$
\begin{aligned}
f^{\prime}(t) & =(d-k) c_{1}^{(d-k-1)} c_{2}^{k}(2 k \beta)+k c_{1}^{(d-k)} c_{2}^{(k-1)} 2(k \beta-d) \\
& =2 k c_{1}^{(d-k-1)} c_{2}^{(k-1)}\left\{\left[d \beta c_{2}+k \beta c_{1}\right]-\left[k \beta c_{2}+d c_{1}\right]\right\}=0
\end{aligned}
$$

onde $c_{1}=(1+2 t k \beta)$ e $c_{2}=(1+2 t(k \beta-d))$. Novamente, $2 k c_{1}^{(d-k-1)} c_{2}^{(k-1)} \neq 0$.

$\operatorname{Logo}\left[d \beta c_{2}+k \beta c_{1}\right]-\left[k \beta c_{2}+d c_{1}\right]=0$. Obtemos assim o ponto crítico

$$
t_{0}=-\frac{1-\beta}{2 \beta(d-k \beta)} \in(0,1 / 2(d-k \beta))
$$

O argumento para provar que $t_{0}$ maximiza a função $f$ é análogo ao item anterior. Assim, ao substituir $t_{0}$ em $g\left(t_{0}\right)=\frac{1}{\sqrt{f\left(t_{0}\right)}}$, temos

$$
\operatorname{Pr}\left[L \geq \beta \frac{k}{d}\right] \leq g\left(t_{0}\right)=\beta^{k / 2}\left(\frac{d-k \beta}{d-k}\right)^{(d-k) / 2} .
$$

A restrição $\beta<d / k$ implica que $\frac{(1-\beta) k}{(d-k)}>-1$. Logo, a desigualdade $\beta^{k / 2}\left(\frac{d-k \beta}{d-k}\right)^{(d-k) / 2} \leq \exp \left(\frac{k}{2}(1-\beta+\ln \beta)\right)$ também é igual ao item anterior. Logo,

$$
\operatorname{Pr}\left[L \geq \beta \frac{k}{d}\right] \leq \beta^{\frac{k}{2}}\left(1+\frac{(1-\beta) k}{(d-k)}\right)^{\frac{(d-k)}{2}} \leq \exp \left(\frac{k}{2}(1-\beta+\ln \beta)\right) .
$$

Concluindo o segundo item.

Fixe $s \in S^{d-1}$ qualquer. Defina a variável aleatória $\mathcal{L}: G_{k}\left(\mathbb{R}^{d}\right) \rightarrow \mathbb{R}$, $E \mapsto\left\|\pi_{E}(s)\right\|^{2}$, A distribuição de $\mathcal{L}$ na reta é dada por

$$
\operatorname{Pr}[\mathcal{L} \in B]=\xi\left\{E \in G_{k}\left(\mathbb{R}^{d}\right):\left\|\pi_{E}(s)\right\|^{2} \in B\right\}, \quad \forall B \in \mathcal{B}(\mathbb{R})
$$


Fato: $\forall s \in S^{d-1}$ e $\forall g \in \mathcal{O}(d)$, temos

$$
\left\|\pi_{g \mathbb{R}^{k}}(s)\right\|=\left\|\pi_{\mathbb{R}^{k}}\left(g^{-1} s\right)\right\|
$$

Demonstração: Sejam $\left\{e_{1}, \cdots, e_{k}\right\}$ a base canônica de $\mathbb{R}^{k}$ e $\left\{g e_{1}, \cdots, g e_{k}\right\}$ uma base ortonormal de $g \mathbb{R}^{k}$. Assim, observamos que $\pi_{g \mathbb{R}^{k}}(s)=\sum_{i=1}^{k}\left\langle s, g e_{i}\right\rangle g e_{i}$ e $\pi_{\mathbb{R}^{k}}\left(g^{-1} s\right)=\sum_{i=1}^{k}\left\langle\left(g^{-1} s\right), e_{i}\right\rangle e_{i}$. Logo, pelo teorema de Pitágoras, temos

$$
\left\|\pi_{g \mathbb{R}^{k}}(s)\right\|^{2}=\sum_{i=1}^{k}\left\langle s, g e_{i}\right\rangle^{2} \quad \text { e } \quad\left\|\pi_{\mathbb{R}^{k}}\left(g^{-1} s\right)\right\|^{2}=\sum_{i=1}^{k}\left\langle g^{-1} s, e_{i}\right\rangle^{2} .
$$

Além disso, $\left\langle s, g e_{i}\right\rangle=\left\langle g^{*} s, g e_{i}\right\rangle=\left\langle g^{-1} s, e_{i}\right\rangle$. Portanto, concluímos que $\left\|\pi_{g \mathbb{R}^{k}}(s)\right\|^{2}=\left\|\pi_{\mathbb{R}^{k}}\left(g^{-1} s\right)\right\|^{2}$.

Teorema 5.7 A variável aleatória $L=\|Z\|^{2}$, definida no Lema 5.6, possui a mesma distribuição de $\mathcal{L}$.

Demonstração: Seja $B \in \mathcal{B}(\mathbb{R})$ um boreliano da reta. Por definição temos que $\operatorname{Pr}[L \in B]=\sigma\left\{x \in S^{d-1}:\left\|\pi_{\mathbb{R}^{k}}(x)\right\|^{2} \in B\right\}^{3}$. Por outro lado, $\sigma$ é o push-forward da medida de Haar do grupo $\mathcal{O}(d)^{4}$, portanto

$$
\sigma\left\{x \in S^{d-1}:\left\|\pi_{\mathbb{R}^{k}}(x)\right\|^{2} \in B\right\}=\mu\left\{g \in \mathcal{O}(d):\left\|g s_{\mathbb{R}^{k}}\right\|^{2} \in B\right\}
$$

Além disso, a igualdade 5-3, implica que

$$
\sigma\left\{x \in S^{d-1}:\left\|\pi_{\mathbb{R}^{k}}(x)\right\|^{2} \in B\right\}=\mu\left\{g \in \mathcal{O}(d):\left\|\pi_{\mathbb{R}^{k}}\left(g^{-1} s\right)\right\|^{2} \in B\right\}
$$

Assim, concluímos que

$$
\operatorname{Pr}[L \in B]=\mu\left\{g \in \mathcal{O}(d):\left\|\pi_{\mathbb{R}^{k}}\left(g^{-1} s\right)\right\|^{2} \in B\right\}
$$

Logo,

$$
\begin{aligned}
& \operatorname{Pr}[\mathcal{L} \in B] \stackrel{(5-10)}{=} \xi\left\{E \in G_{k}\left(\mathbb{R}^{d}\right):\left\|\pi_{E}(s)\right\|^{2} \in B\right\} \\
& (5=4) \quad \mu\left\{g \in \mathcal{O}(d):\left\|\pi_{g \mathbb{R}^{k}}(s)\right\|^{2} \in B\right\} \\
& \stackrel{(5=11)}{=} \mu\left\{g \in \mathcal{O}(d):\left\|\pi_{\mathbb{R}^{k}}\left(g^{-1} s\right)\right\|^{2} \in B\right\} \stackrel{(5-12)}{=} \operatorname{Pr}[L \in B] .
\end{aligned}
$$

O corolário a seguir é uma consequência direta do Teorema 5.7 e do Lema 5.6.

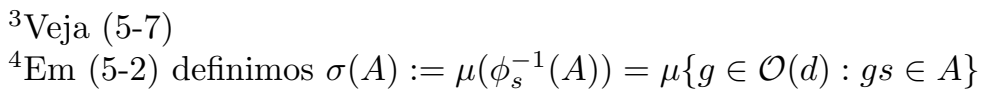


Corolário 5.8 Seja $k<d$. Temos então:

a. Se $0<\beta<1$, então

$$
\operatorname{Pr}\left[\mathcal{L} \leq \frac{\beta k}{d}\right] \leq \exp \left(\frac{k}{2}(1-\beta+\ln \beta)\right)
$$

b. Se $1<\beta<d / k$, então

$$
\operatorname{Pr}\left[\mathcal{L} \geq \frac{\beta k}{d}\right] \leq \exp \left(\frac{k}{2}(1-\beta+\ln \beta)\right) .
$$

\section{4}

\section{Demonstração do teorema de Johnson-Lindenstrauss}

Teorema. (Johnson-Lindenstrauss) Fixe $\epsilon \in(0,1)$ e $d, n \in \mathbb{N}$. Tome $k \in \mathbb{N}$ tal que

$$
d>k \geq 4\left(\frac{\epsilon^{2}}{2}-\frac{\epsilon^{3}}{3}\right)^{-1} \ln n .
$$

Então, para qualquer conjunto $V \operatorname{com} n$ pontos em $\mathbb{R}^{d}$, existe um subespaço $E \subset \mathbb{R}^{d}$ de dimensão $k$ para o qual temos que $\forall u, v \in V$

$$
(1-\epsilon)\|u-v\|^{2} \leq\left\|\sqrt{d / k}\left(\pi_{E}(u)-\pi_{E}(v)\right)\right\|^{2} \leq(1+\epsilon)\|u-v\|^{2},
$$

onde $\pi_{E}: \mathbb{R}^{d} \rightarrow E$ é a projeção ortogonal sobre $E$.

Demonstração: Para cada $E \in G_{k}\left(\mathbb{R}^{d}\right)$ considere a projeção $\pi_{E}: \mathbb{R}^{d} \rightarrow E$, $x \mapsto \pi_{E}(x)$. Fixamos $u, v \in V$ e fazemos $s=\frac{u-v}{\|u-v\|} \in S^{d-1}$. Assim, definimos a variável aleatória $\mathcal{L}: G_{k}\left(\mathbb{R}^{d}\right) \rightarrow \mathbb{R}, E \mapsto\left\|\pi_{E}(s)\right\|^{2}, \pi_{E}(s)=\frac{\pi_{E}(u)-\pi_{E}(v)}{\|u-v\|}$.

Tomamos $\beta_{1}=1-\epsilon$ e aplicamos o item $a$ do Corolário 5.8

$$
\begin{aligned}
\xi\left[\frac{\left\|\sqrt{d / k}\left(\pi_{E}(u)-\pi_{E}(v)\right)\right\|^{2}}{\|u-v\|^{2}} \leq \beta\right] & =\operatorname{Pr}\left[\mathcal{L} \leq \frac{\beta k}{d}\right] \leq \exp \left(\frac{k}{2}(1-\beta+\ln \beta)\right) \\
& =\exp \left(\frac{k}{2}(1-(1-\epsilon)+\ln (1-\epsilon))\right) \\
& =\exp \left(\frac{k}{2}(\epsilon+\ln (1-\epsilon))\right) .
\end{aligned}
$$

De $\ln (1+x)=\sum_{n=1}^{\infty} \frac{(-1)^{n+1}}{n} x^{n}$, concluímos que $\ln (1-x) \leq-x-\frac{x^{2}}{2}$ para todo $0 \leq x<1$. Logo, exp $\left(\frac{k}{2}(\epsilon+\ln (1-\epsilon))\right) \leq \exp \left(\frac{k}{2}\left(\epsilon-\epsilon-\frac{\epsilon^{2}}{2}\right)\right)=\exp \left(\frac{-k \epsilon^{2}}{4}\right)$. Manipulando a hipótese $k \geq 4\left(\frac{\epsilon^{2}}{2}-\frac{\epsilon^{3}}{3}\right)^{-1} \ln n$, obtemos $\frac{-k \epsilon^{2}}{4} \leq-2 \ln n$. Logo, 


$$
\xi\left[\frac{\left\|\sqrt{d / k}\left(\pi_{E}(u)-\pi_{E}(v)\right)\right\|^{2}}{\|u-v\|^{2}} \leq \beta\right] \leq \exp \left(\ln n^{-2}\right)=\frac{1}{n^{2}}
$$

Analogamente, se tomamos $\beta_{2}=1+\epsilon$ e aplicamos o item $b$ do Corolário 5.8, temos

$$
\begin{aligned}
\xi\left[\frac{\left\|\sqrt{d / k}\left(\pi_{E}(u)-\pi_{E}(v)\right)\right\|^{2}}{\|u-v\|^{2}} \geq \beta\right] & =\operatorname{Pr}\left[\mathcal{L} \geq \frac{\beta k}{d}\right] \leq \exp \left(\frac{k}{2}\left(1-\beta_{2}+\ln \beta_{2}\right)\right) \\
& =\exp \left(\frac{k}{2}(1-(1+\epsilon)+\ln (1+\epsilon))\right) \\
& =\exp \left(\frac{k}{2}(-\epsilon+\ln (1+\epsilon))\right) .
\end{aligned}
$$

Por Taylor, $\ln (1+x) \leq x-\frac{x^{2}}{2}+\frac{x^{3}}{3}$ para todo $x \geq 0$. Logo, $\exp \left(\frac{k}{2}(-\epsilon+\ln (1+\epsilon))\right) \leq \exp \left(\frac{k}{2}\left(-\epsilon+\epsilon-\frac{\epsilon^{2}}{2}+\frac{\epsilon^{3}}{3}\right)\right)=\exp \left(-\frac{k}{2}\left(\frac{\epsilon^{2}}{2}-\frac{\epsilon^{3}}{3}\right)\right)$. Novamente, manipulando $k \geq 4\left(\frac{\epsilon^{2}}{2}-\frac{\epsilon^{3}}{3}\right)^{-1} \ln n$, temos $-\frac{k}{2}\left(\frac{\epsilon^{2}}{2}-\frac{\epsilon^{3}}{3}\right) \leq-2 \ln n$. Logo,

$$
\xi\left[\frac{\left\|\sqrt{d / k}\left(\pi_{E}(u)-\pi_{E}(v)\right)\right\|^{2}}{\|u-v\|^{2}} \geq \beta\right] \leq \exp \left(\ln n^{-2}\right)=\frac{1}{n^{2}}
$$

Assim,

$$
\xi\left\{E \in G_{k}\left(\mathbb{R}^{d}\right): \frac{\left\|\sqrt{d / k}\left(\pi_{E}(u)-\pi_{E}(v)\right)\right\|^{2}}{\|u-v\|^{2}} \notin(1-\epsilon, 1+\epsilon)\right\} \leq \frac{2}{n^{2}} .
$$

Portanto, a probabilidade de $\frac{\left\|\sqrt{d / k}\left(\pi_{E}(u)-\pi_{E}(v)\right)\right\|^{2}}{\|u-v\|^{2}}$ não pertencer ao intervalo $(1-\epsilon, 1+\epsilon)$ para dois pontos quaisquer $u, v \in V$ é menor ou igual a

$$
\left(\begin{array}{l}
n \\
2
\end{array}\right) \times \frac{2}{n^{2}}=\frac{n !}{(n-2) ! 2} \times \frac{2}{n^{2}}=\frac{n(n-1)}{n^{2}}=1-\frac{1}{n} .
$$

Logo, concluímos que para qualquer para de vetores $u, v \in V$, temos

$$
\begin{aligned}
& \xi\left\{E \in G_{k}\left(\mathbb{R}^{d}\right):(1-\epsilon) \leq \frac{\left\|\sqrt{d / k}\left(\pi_{E}(u)-\pi_{E}(v)\right)\right\|^{2}}{\|u-v\|^{2}} \leq(1+\epsilon)\right\} \\
= & 1-\xi\left\{E \in G_{k}\left(\mathbb{R}^{d}\right): \frac{\left\|\sqrt{d / k}\left(\pi_{E}(u)-\pi_{E}(v)\right)\right\|^{2}}{\|u-v\|^{2}} \notin(1-\epsilon, 1+\epsilon)\right\} \\
\geq & \frac{1}{n} .
\end{aligned}
$$


Ou seja, dadas todas as condições suficientes do teorema, existe $\pi_{E}: \mathbb{R}^{d} \rightarrow E$ tal que

$$
(1-\epsilon)\|u-v\|^{2} \leq\left\|\sqrt{d / k}\left(\pi_{E}(u)-\pi_{E}(v)\right)\right\|^{2} \leq(1+\epsilon)\|u-v\|^{2} .
$$




\section{A Noções de Variedades}

Lembramos para conveniência do leitor o vocabulário básico associado à construção de variedades. Seja $M$ um conjunto qualquer. Uma carta local $(U, \varphi)$ é uma bijeção $\varphi: U \rightarrow \varphi(U)$ de um subconjunto $U \subset M$ sobre um aberto $\varphi(U) \subset \mathbb{R}^{m}$. Um atlas $\mathfrak{A}$ de dimensão $m$ e diferenciável de classe $C^{k}$ $(k>1)$ sobre $M$ é uma família de cartas locais $\left\{\left(U_{i}, \varphi_{i}\right): i \in I, \varphi_{i}\left(U_{i}\right) \subset \mathbb{R}^{m}\right\}$ que cobrem $M$, no sentido que $M=\cup_{i \in I} U_{i}$ e para as quais todas as mudanças de coordenadas são de classe $C^{k}$. Ou seja, se $\left(U_{i}, \varphi_{i}\right)$ e $\left(U_{j}, \varphi_{j}\right)$ são duas cartas de $\mathfrak{A}$ com $U_{i} \cap U_{j} \neq \emptyset$, então a aplicação

$$
\varphi_{j} \circ \varphi_{i}^{-1}: \varphi_{i}\left(U_{i} \cap U_{j}\right) \rightarrow \varphi_{j}\left(U_{i} \cap U_{j}\right)
$$

é um difeomorfismo de classe $C^{k}$.

Uma carta local $(U, \varphi)$ é admissivel a um atlas $\mathfrak{A}$ se $\mathfrak{A} \cup(U, \varphi)$ ainda é um atlas de classe $C^{k}$ em M.

Uma variedade diferenciável de dimensão $m$ e classe $C^{k}$ é um par ordenado $(M, \mathfrak{A})$ onde $M$ é um conjunto e $\mathfrak{A}$ é um atlas de dimensão $m$ e de classe $C^{k}$ sobre $M$. Por praticidade escrevemos variedade diferenciável $M$ ou simplesmente variedade $M$ quando o atlas for fácil de identificar. Quando a estrutura é $C^{\infty}$, diremos que a variedade é suave.

Seja $M$ uma variedade diferenciável $M$. Um subconjunto $A \subset M$ é aberto se para cada $a \in A$ existe uma carta local admissível $(U, \varphi)$ tal que $a \in U$ e $U \subset A$. Desta maneira, induzimos uma topologia à variedade $M$.

Seja $(M, \mathfrak{A})$ uma variedade de dimensão $m$. Um subconjunto $N \subset M$ é uma subvariedade de $M$ e dimensão $n \leq m$ se para todo $x \in N$ existe uma carta local $(U, \varphi)$ admissível ao atlas $\mathfrak{A} \operatorname{com} x \in U$ e tal que $\varphi: U \rightarrow \mathbb{R}^{n} \times \mathbb{R}^{m-n}$ satisfaz $\varphi(U \cap N)=\varphi(U) \cap\left(\mathbb{R}^{n} \times\{0\}\right)$.

Seja $U_{0} \subset \mathbb{R}^{m}$ um aberto. Uma aplicação diferenciável $f: U_{0} \rightarrow \mathbb{R}^{m+n}$ é uma imersão quando, para cada $x \in U_{0}, f^{\prime}(x): \mathbb{R}^{m} \rightarrow \mathbb{R}^{m+n}$ é injetiva. Uma parametrização de classe $C^{k}$ e dimensão $m$ de um conjunto $U \subset \mathbb{R}^{n}$ é uma imersão $\varphi: U_{0} \rightarrow U$ de classe $C^{k}$ que é, ao mesmo tempo, um homeomorfismo do aberto $U_{0} \subset \mathbb{R}^{m}$ sobre $U$. 
Um conjunto $M \subset \mathbb{R}^{n}$ chama-se uma superfície de dimensão $m$ e classe $C^{k}$ quando todo ponto $p \in M$ está contido em algum aberto $U \subset \mathbb{R}^{n}$ de maneira que $V=U \cap M$ é imagem de uma parametrização $\varphi: V_{0} \subset \mathbb{R}^{m} \rightarrow V$, de dimensão $m$ e classe $C^{k}$. 


\section{B \\ Noções de Probabilidade}

Um espaço de probabilidade é um espaço de medida $(\Omega, \mathcal{A}, \xi)$ onde $\mathcal{A}$ é uma $\sigma$-álgebra de eventos aleatórios e $\xi$ é uma medida de probabilidade, ou seja, $\xi(X)=1$. Uma variável aleatória em um espaço de probabilidade $(\Omega, \mathcal{A}, \xi)$ é uma função real $X: \Omega \rightarrow \mathbb{R}$ tal que $[X \in B] \in \mathcal{A}, \forall B \in \mathcal{B}(\mathbb{R})$. Ou seja, $[X \in B]$ é um evento aleatório para todo boreliano $B \subset \mathbb{R}$. Se $f: \mathbb{R} \rightarrow \mathbb{R}$ é uma função mensurável, então $X \circ f$ é ainda uma variável aleatória.

Se $X$ é uma variável aleatória em um espaço de medida $(\Omega, \mathcal{A}, \xi)$, então a medida de probabilidade nos borelianos de $\mathbb{R}$ dada por

$$
\operatorname{Pr}(B)=\xi\{\omega \in \Omega: X(\omega) \in B\}, \quad B \in \mathcal{B}(\mathbb{R})
$$

é chamada de distribuição de probabilidade ou simplesmente distribuição de $X$ em $(\mathbb{R}, \mathcal{B}(\mathbb{R}))$. Um vetor $X=\left(X_{1}, X_{2}, \cdots, X_{d}\right)$ onde cada coordenada é uma variável aleatória e todas elas definidas no mesmo espaço de probabilidade $(\Omega, \mathcal{A}, \xi)$ é chamado de vetor aleatório. Analogamente, definimos a distribuição de um vetor aleatório $X=\left(X_{1}, X_{2}, \cdots, X_{d}\right)$ como

$$
\operatorname{Pr}(B)=\{\omega \in \Omega: X(\omega) \in B\}, \quad B \in \mathcal{B}\left(\mathbb{R}^{d}\right) .
$$

Seja $X$ uma variável aleatória definida no espaço de medida $(\Omega, \mathcal{A}, \xi)$. Na linguagem de teoria da medida, $X$ é uma função mensurável e a esperança $X$ é definida como sendo a integral com respeito à medida $\xi$. A esperança de $X$ é denotada como

$$
\mathbb{E}[X]:=\int_{\Omega} X d \xi .
$$

Se $X$ é uma variável aleatória não negativa e $a>0$, então a desigualdade de Markov afirma que

$$
\operatorname{Pr}[X \geq a] \leq \frac{\mathbb{E}[X]}{a} .
$$

Terminamos este apêndice como a seguinte proposição:

Proposição B.0.1 Se a variável aleatória $X$ tem distribuição normal univariada, então para $-\infty<s<1 / 2$, temos $\mathbb{E}\left[\exp \left(s X^{2}\right)\right]=1 / \sqrt{1-2 s}$. 
Demonstração: Chame de $\alpha=\frac{1}{\sqrt{2 \pi}}$

$$
\begin{aligned}
\mathbb{E}\left[\exp \left(s X^{2}\right)\right] & =\alpha \int_{\mathbb{R}} \exp \left(s t^{2}\right) \exp \left(-t^{2} / 2\right) d t=\alpha \int_{\mathbb{R}} \exp \left(\left(2 s t^{2}-t^{2}\right) / 2\right) d t \\
& =\alpha \int_{\mathbb{R}} \exp \left((t(\sqrt{1-2 s}))^{2} / 2\right) d t .
\end{aligned}
$$

Fazendo $z=t(\sqrt{1-2 s})$ e assumindo $-\infty<s<1 / 2$, concluímos que

$$
\mathbb{E}\left[\exp \left(s X^{2}\right)\right]=\frac{1}{\sqrt{1-2 s}} \frac{1}{\sqrt{2 \pi}} \int_{\mathbb{R}} \exp \left(z^{2} / 2\right) d z=1 / \sqrt{1-2 s}
$$




\section{Referências Bibliográficas}

[1] ABRAham, R.; MARSDEN, J. E.; RATIU, T. S. ; CUSHMAN, R.. Foundations of mechanics. Benjamin/Cummings Publishing Company, 1978.

[2] BARTLE, R. G.; BARTLE, R. G.. The elements of integration and Lebesgue measure. Wiley Online Library, 1995.

[3] DASGUPTA, S.; GUPTA, A.. An elementary proof of a theorem of johnson and lindenstrauss. Random Structures \& Algorithms, 22(1):60-65, 2002.

[4] FOLLAND, G. B.; FOLLAND, G.. Real analysis: modern techniques and their applications, volumen 2. Wiley New York, 1984.

[5] HERSTEIN, I. N.; HERSTEIN, I.. Abstract algebra, volumen 21990. Macmillan New York, 1986.

[6] JAMES, B. R.. Probabilidade: Um curso em nível intermediário, coleção euclides. Rio de Janeiro. IMPA, 2a Edição, 1996.

[7] KRANTZ, S. G.; PARKS, H. R.. Geometric integration theory. Birkhäuser Boston, 2008.

[8] LEDOUX, M.. The concentration of measure phenomenon, volumen 89. Amer Mathematical Society, 2001.

[9] LIMA, E. L.. Algebra linear, sétima ediçao. SBM, Coleçao Matemática Universitária, 2004.

[10] LIMA, E. L.. Curso de análise vol. 2 (oitava ediçao). Projeto Euclides. IMPA, 2005.

[11] LIMA, E. L.. Variedades diferenciáveis. Publicações Matemáticas, 2007.

[12] MUNKRES, J. R.. Topology. Prentice Hall, 2nd edition, 2000.

[13] ROYDEN, H.. Real analysis. Macmillan New York, 1968. 
[14] SHIRYAEV, A.. Probability. number 95 in graduate texts in mathematics, 1996.

[15] TREFEthen, L. N.; BAU III, D.. Numerical linear algebra. Número 50. Society for Industrial Mathematics, 1997. 